\title{
INTS13 Mutations Causing a Developmental Ciliopathy Disrupt Integrator Complex Assembly
}

3

1

Lauren G. Mascibroda ${ }^{1, *}$, Mohammad Shboul, ${ }^{2,}{ }^{*}$ Nathan D. Elrod ${ }^{1}$, Laurence Colleaux ${ }^{3}$, Hanan Hamamy $^{4}$, Kai-Lieh Huang ${ }^{1}$, Natoya Peart ${ }^{1}$, Moirangthem Kiran Singh ${ }^{1}$, Hane Lee ${ }^{5}$, Barry Merriman $^{5}$, Jeanne N. Jodoin ${ }^{6}$, Laura A. Lee ${ }^{7}$, Raja Fathalla ${ }^{2}$, Baeth Al-Rawashdeh ${ }^{8}$, Osama Ababneh $^{8}$, Mohammad El-Khateeb ${ }^{9}$, Nathalie Escande-Beillard ${ }^{13}$, Stanley F. Nelson ${ }^{5}$, Yixuan $\mathrm{Wu}^{10}$, Liang Tong ${ }^{10}$, Linda J. Kenney ${ }^{1}$, William K. Russell ${ }^{1}$, Jeanne Amiel ${ }^{11}$, Bruno Reversade ${ }^{12}$, $13,14,15^{* *}$, and Eric J. Wagner ${ }^{1, * *}$

${ }^{1}$ Department of Biochemistry and Molecular Biology, The University of Texas Medical Branch at Galveston, Galveston TX 77550, USA

${ }^{2}$ Department of Medical Laboratory Sciences, Jordan University of Science and Technology, Irbid, Jordan

${ }^{3}$ Inserm UMR 1163, Institut Imagine, 24 Boulevard du Montparnasse, 75015 Paris, France

${ }^{4}$ Department of Genetic Medicine and Development, University Hospital, Geneva, Switzerland

${ }^{5}$ Department of Pathology and Laboratory Medicine, Department of Human Genetics, David Geffen

School of Medicine, University of California Los Angeles, Los Angeles CA 90095, USA

${ }^{6}$ Department of Cell and Developmental Biology, Vanderbilt University Medical Center,

Nashville, TN 37232, USA

${ }^{7}$ Department of Pathology, Microbiology and Immunology, Vanderbilt University Medical Center, Nashville, TN 37232, USA

${ }^{8}$ Faculty of Medicine, Hospital of the University of Jordan, University of Jordan, Jordan

${ }^{9}$ National Center for Diabetes, Endocrinology and Genetics, Jordan

${ }^{10}$ Department of Biological Sciences, Columbia University, New York, NY 10027, USA

${ }^{11}$ Service de Génétique, Institut Imagine, 24 Boulevard du Montparnasse 75015, Paris, France

${ }^{12}$ Department of Paediatrics, School of Medicine, NUS, Singapore

${ }^{13}$ Department of Medical Genetics, KOÇ University, Istanbul, Turkey

${ }^{14}$ Genome Institute of Singapore, A*STAR, 137673, Singapore

${ }^{15}$ Institute of Molecular and Cell Biology, A*STAR, Singapore

* These authors contributed equally to this work

** Corresponding authors: bruno@,reversade.com and ejwagner@utmb.edu 
37 Oral-facial-digital syndromes (OFD) are a heterogeneous group of congenital 38 disorders characterized by malformations of the face and oral cavity, and digit anomalies. To date, mutations in 12 ciliary-related genes have been identified that cause several types of OFD, suggesting that OFDs constitute a subgroup of developmental ciliopathies. Through homozygosity mapping and exome sequencing of two families with variable OFD type 2, we identified distinct germline mutations in INTS13, a subunit of the Integrator complex. This 14-component complex associates with RNAPII and can cleave nascent RNA to modulate gene expression. We determined that INTS13 utilizes a discrete domain within its Cterminus to bind the Integrator cleavage module, which is disrupted by the identified germline INTS13 mutations. Depletion of INTS13 disrupts ciliogenesis in human cultured cells and causes dysregulation of a broad collection of ciliary genes. Accordingly, its knockdown in Xenopus embryos lead to motile cilia anomalies. Altogether, we show that mutations in INTS13 cause an autosomal recessive ciliopathy, which reveals key interactions within Integrator components. 


\section{INTRODUCTION}

64

Ciliopathies encompass a large and expanding group of human genetic disorders caused by mutations in genes either encoding ciliary proteins or that participate indirectly in the formation and function of cilia1, 2, 3, 4 . There have been several hundred diseases identified as ciliopathies that range from renal cystic disease ${ }^{5}$ and isolated blindness ${ }^{6}$ to multiple organ disorders such as a Bardet-Biedl syndrome $(B B S)^{7}$ or Meckel Gruber syndrome $(\mathrm{MKS})^{8}$. The clinical manifestations associated with ciliopathies are broad and highly variable and include: retinal degeneration, specific nervous system malformations, hepatic, kidney and pancreatic cysts, heterotaxia, polydactyly, encephalocele, hydrocephalus, hearing loss, anosmia, intellectual disability, developmental delay, skeletal malformation, craniofacial anomalies, respiratory function impairment, infertility and obesity $4,9,10,11,12,13$.

Among ciliopathies, oral-facial-digital syndromes (OFDs) are a group of clinically and genetically heterogeneous developmental disorders characterized by defects in the development of the face and oral cavity along with digit anomalies. Symptoms associated with OFDs can also be found in other organs, which has led to the classification of at least 14 different types of OFD ${ }^{14,15}$. To date, 12 genes have been identified to be mutated in OFD syndromes and the encoded proteins have been implicated in primary cilia biogenesis and function. The phenotypic spectrum of OFD syndromes can overlap with other known ciliopathies, and mutations in OFD genes can also cause other types of ciliopathies $^{16}, 17,18,19,20,21,22$. Of the 12 known genes involved in OFDs, the majority encode structural components of the primary cilium, such as OFD123 and TMEM23124. However, not all OFD-causing gene mutations such as those in the RNA helicase DDX59 ${ }^{19}$ localize to the primary cilium, and are thus thought to contribute indirectly to disease pathology. Despite significant progress, seven OFD syndromes are still without a clear genetic aetiology and at least three unclassified forms need further clinical and molecular characterization.

The Integrator complex (INT), which is unique to metazoans, is composed of at least 14 subunits, and it associates with RNA polymerase II (RNAPII) where it modulates transcription $25,26,27,28,29$. Critical among its protein components is Integrator subunit 11 
$\left(\right.$ INTS11) ${ }^{30}$, which is a member of the metallo- $\beta$-lactamase/ $\beta$-CASP family of RNA endonucleases ${ }^{31}$ and is a paralog of the cleavage and polyadenylation specificity factor of $73 \mathrm{kDa}$ (CPSF73), which cleaves pre-mRNA ${ }^{32}$. INTS11 has been found to associate with INTS4 and INTS9 to form what is called the Integrator Cleavage Module (CM), which is thought to be the key enzymatic factor within the complex ${ }^{33}$. The Integrator $\mathrm{CM}$, through the activity of INTS11, has been shown to be essential to cleave the 3' ends of a variety of noncoding RNA and nascent mRNA of protein-coding genes with paused RNAPII. This catalytic activity thus serves as a key functional component of Integrator-mediated transcriptional repression and for the biogenesis of noncoding $\operatorname{RNA}^{34,35,36,37,38 .}$

The potential connection between Integrator function and ciliogenesis was uncovered initially in Drosophila, where depletion of nearly any Integrator subunit disrupts proper centriole localization, an early event in ciliogenesis ${ }^{39}, 40$. Further evidence was attained using human retinal pigment epithelial (RPE) cells, where depletion of multiple Integrator subunits can lead to inhibition of primary cilium biogenesis ${ }^{41}$. More recently, an unbiased CRISPR screen for factors required for ciliogenesis also identified several Integrator subunits as essential to form primary cilia ${ }^{42}$. Collectively, these data imply that the overall function of the Integrator complex, and not any specific subunit, is required for proper cilia formation. Given its role in modulating RNAPII behavior at diverse gene types, the precise function for Integrator in ciliogenesis is most likely at the level of gene expression. However, the ciliary genes subject to Integrator regulation are currently unknown, and no ciliopathies have been found associated with Integrator subunit mutations.

Here, we used a combination of homozygosity mapping and deep sequencing of patient samples to identify two distinct recessive germline mutations in the INTS13 (MIM615079) gene causing a variable form of OFD syndrome type 2 (OFD2), also known as Mohr Syndrome (MIM252100 $)^{43}$. Using multiple molecular and biochemical approaches, we demonstrate that the C-terminus of INTS13 interacts with the Integrator $\mathrm{CM}$ and that mutations observed in OFD2 patients specifically disrupt this interaction. We also find that human cells depleted of INTS13 not only have reduced primary cilia, but also exhibit broad transcriptional dysregulation that impacts numerous known genes required for proper ciliogenesis. These observations were validated in vivo, where 
125

126

127

128

129

130

131

132

133

134

135

136

137

138

139

140

141

142

143

144

145

146

147

148

149

150

151

152

153

154

155

morpholino-mediated depletion of ints13 in Xenopus laevis leads to profound underdevelopment, with a specific reduction in the formation and beating of multiciliated cells. Taken together, these results reveal a key role for Integrator in human ciliogenesis and identify a vital interaction between two Integrator subcomplexes.

\section{RESULTS}

\section{Characterization of 4 patients with recessive loss-of-function INTS13 mutations}

In Family 1, two affected sisters (individuals II.4 and II.5 in Figure 1a) born to a consanguineous Jordanian couple presented with a congenital disorder consistent with OFD2 (MIM252100). The two sisters displayed similar phenotypes encompassing craniofacial dysmorphisms, including: a bilateral cleft lip $(C L)$, microcephaly, hypertelorism, epicanthal fold, broad nasal root with depressed nasal tip, large and low set ears, long philtrum and thick septum. In addition to bilateral $\mathrm{CL}$, their oral cavity had omega shape epiglottis, underdeveloped epiglottic folds and hypertrophied false vocal cords. Dental abnormalities were also observed, such as central median upper incisor in II.4 and single central upper incisor in II.5. Supernumerary teeth were observed in II.4. Both sisters have clinodactyly of the $4^{\text {th }}$ and $5^{\text {th }}$ fingers and toes, and pre-axial polydactyly was observed in one hand of II.4. Single transverse palmar creases on both hands and rough hair were also observed in both sisters. Audiologic examination of the two sibs revealed the presence of tympanic membrane retraction and calcification (myringosclerosis). Ophthalmic examination revealed dilated retinal vessels and a crowded optic disk. Patients were reported to have chronic urinary tract infection and recurrent respiratory tract infections with chronic cough. Supravalvular pulmonary stenosis and partial anomalous pulmonary venous return were reported in patient II.5 soon after birth. Taken together, both patients share the cardinal features of an OralFacial-Digital Syndrome and exhibit a wide range of clinical manifestations. The clinical findings of these patients are illustrated in Supplemental Figure 1a-m, and a summary is provided in Table 1. Inter- and intra-familial variations have been reported in OFD and were clearly observed between the two sisters. Many clinical features are unique to this 
156 family and overlap with OFD2 or may be a new form of OFD, with a distinct genetic aetiology.

To identify the genetic origin of this developmental disorder, the parents, both affected siblings, and healthy siblings were screened on a 610k Illumina SNP array. A subsequent homozygosity identical-by-descent (IBD) mapping revealed five homozygous candidate regions that were unique to the proposita, with a total size of $45.76 \mathrm{Mb}$ on chromosomes 6, 8, 12, 13 and 16 (Figure 1b). We next employed targeted genomic loci capture followed by massive parallel sequencing to screen 395 candidate genes in these loci $^{44}$. Out of 894 mismatches, one homozygous missense mutation (c.6585A>T) in SACS (MIM604490) and a frameshift (c.2004delA) mutation in INTS13 (MIM615079) were found in the two affected sisters. The mutation in SACS was ruled out as the likely cause due to the gene's known role in causing spastic ataxia (MIM 604490), which did not align with the patients' phenotypes ${ }^{45,46}$. The single base pair (bp) deletion in exon 16 of INTS13 was never observed in GnomAD and is predicted to be damaging according to SIFT or MutationTaster. It alters the reading frame, leading to a premature termination codon (PTC) at p.K668Nfs ${ }^{*} 9$ and the deletion of the remaining 31 amino acids of the INTS13 protein (Figure 1c). This private allele segregated with the disease in all family members available (Supplemental Figure 1n).

The second family had two affected siblings born to French parents not known to be endogamous. The affected 27-year-old woman (II.2) and her affected 20-year-old brother (II.4) (Family 2, Figure 1a) presented with some overlapping phenotypes, but also distinct features from those reported in the Jordanian patients. Both affected sibs were born at term with low birth parameters for weight, length and occipitofrontal circumference (OFC). II.4 presented with hypospadias. Both remained short and became overweight from the age of 3 years, while OFC was in the low normal range at this age. II.2 walked unaided before 18 months and II.4 walked at 22 months. For both, language was delayed and remained limited to a few spoken words, although they could understand simple conversational topics. II.2 was treated for early-onset puberty for 18 months starting at 8 years. Both presented with short extremities with brachydactyly of fingers and toes, 185 hyperlordosis when standing, flessum of the knees and pes valgus. Facial features include upslanting palpebral fissures, macrostomia, gum hypertrophy and a bulbous nasal 
187 tip. They wear glasses for correction of hyperopia and astigmatism. At 20 years, 188 regression with less social interactions, vocal stereotypies and tip toe walking were noted in II.4, while his brain CT-scan showed some cerebellar atrophy and calcification of the cerebellum tent. II.2 has remained stable with no regression observed thus far. The clinical findings of these patients are illustrated in Supplemental Figure 10-w, and a summary is provided in Table 1.

Agilent 60k Array CGH showed no disequilibrium in either sibling. Exome capture was performed followed by sequencing of both affected siblings and their healthy parents. Each sample had an overall mean depth of coverage greater than 90X and more than $97 \%$ of the exome covered at least 15X. Filtering for germline compound heterozygous variants, X-linked or shared heterozygous de novo variants did not provide any candidate; however, a single shared homozygous variant in INTS13 (NM_018164.2: c.1955C>T) emerged as the only candidate mutation (Figure 1c). This mutation converts Serine652 to a Leucine and is located 16 amino acids upstream of where the frameshift mutation was observed in Family 1.

INTS13 mRNA consists of 17 exons (Figure 1c). Its encoded protein is 706 amino acid residues long and was initially termed Mat89Bb (Maternal transcript 89Bb) due to its maternal expression in Drosophila embryos ${ }^{47,48}$. Mat89b was later changed to Asunder/ASUN given its mutant phenotype of altered association of the nuclei with asters and disrupted spermatogenesis ${ }^{49}$. Importantly, ASUN was later shown to be a component of the Integrator complex, although little is known of its distinct function in that context ${ }^{50}$. There is an overall high degree of sequence similarity within the C-termini of INTS13 proteins found in multiple organisms, and both mutated residues are highly conserved between vertebrate and invertebrate species (Figure 1d), suggesting that alteration of these amino acids may lead to a pathogenic function of the INTS13 protein.

To investigate the impact of INTS13 mutations on its expression, we isolated primary cutaneous fibroblasts from affected patients in both families as well as fibroblasts from healthy individuals. We first compared mRNA expression levels by RT-qPCR of INTS13 in Family 1 cells (F1) containing the INTS13 truncation mutation. We found the level of INTS13 transcripts to be significantly down-regulated (Figure 2a), which we 
hypothesized may be due to the presence of the premature termination codon triggering nonsense-mediated decay (NMD). Therefore, we isolated total RNA from patient cells that were either mock-treated or treated with cycloheximide, a translational inhibitor that blocks NMD, and RT-qPCR was repeated. The results showed that the level of mutant mRNA was increased following cycloheximide treatment, suggesting that the mutant INTS13 mRNA was indeed targeted for NMD (Figure $2 b$ ). To assess the levels of INTS13 protein in $\mathrm{F} 1$ cells, we raised two custom antibodies against antigens from the central portion of INTS13 ( $\alpha-M)$ and the C-terminal region $(\alpha-C)$, which is downstream of the truncation. These antibodies were affinity-purified and used to probe cell lysates from patient fibroblasts using Western blot analysis. We observed that the C-terminal antibody could detect endogenous INTS13 protein in control cells, but not in F1 patient fibroblasts, likely due to the loss of the epitope in the truncated mutant (Figure 2c). Consistent with this notion, we could detect low levels of truncated INTS13 in F1 patient fibroblasts using the $\alpha-M$ antibody (Figure 2c). Overall, these results reveal that the INTS13 mutation observed in Family 1 creates an mRNA subject to NMD that is translated at low levels, thereby producing a truncated INTS13 protein.

We next analyzed the expression of INTS13 in fibroblasts isolated from a Family 2 (F2) patient harboring the homozygous p.S652L mutation. We observed a slight but significant reduction in the level of endogenous INTS13 mRNA in these mutant cells (Figure $2 \mathrm{~d}$ ) and a pronounced reduction in the level of INTS13 protein as assessed using either antibody (Figure 2e). We posited that the basis for the reduction in INTS13 protein, beyond the minor reduction in mRNA, may be due to increased protein degradation of the INTS13 F2 mutant. To test this, primary fibroblasts were treated with MG132 to inhibit proteasome activity, and we observed an increased level of INTS13 protein comparable to control cells by Western blot analysis (Figure 2f). This suggests that the INTS13 mutation observed in Family 2 generates a protein which may be unstable and targeted for degradation via the proteasome.

The epithelia lining the human respiratory system are covered with multiple cell types dominated by ciliated cells. These ciliated cells have hundreds of motile cilia to generate fluid flow that keeps mucus moving to protect the airway from inhaled irritants, pathogens and foreign particles. Abnormalities of mucociliary clearance caused by either 
aberrant ciliated cells or cilia dysfunction often result in recurrent respiratory tract infections. Reduction or impairment of mucociliary clearance in humans results in an array of pathologies such as primary ciliary dyskinesia, chronic obstructive pulmonary disease and asthma $51,52,53,54,55$. In this study, both probands of Family 1 suffered from recurrent respiratory tract infection and chronic cough. Therefore, we obtained respiratory epithelial cells from the two patients and the mother to assess cilia formation and performance. Immunofluorescence staining was performed using anti-acetylated $\alpha$-Tubulin antibody to visualize cilia. The average length of nasal cilia was then scored. The results showed that cilia were shorter, fewer in number and occasionally disorganized compared to those of the heterozygous but unaffected mother, which may explain the recurrent respiratory tract infections in the two affected subjects (Figure $2 g-h$ ). Importantly, these results also indicate that ciliogenesis was indeed disrupted in patient cells.

\section{OFD2 patient mutations within the INTS13 C-terminus disrupt association with the} Integrator Cleavage Module

Recently, the structure of INTS13 associated with INTS14 has been elucidated. However, the INTS13 C-terminus was unable to be resolved in that structure, thereby obscuring our ability to ascertain the impact of OFD2 mutations ${ }^{56}$. The INTS13/14 heterodimer was shown to interact with INTS10 as well as the Integrator CM, the latter of which was found to be mediated independently through the INTS13 C-terminus ${ }^{56}$. With this information in hand, and our previous success mapping Integrator interactions using yeast two-hybrid analysis ${ }^{33,50,57,58}$, we fused INTS13 to the Gal4 DNA binding domain and screened for interactions with the other Integrator subunits that were each expressed individually as Gal4 activation domain fusions. This initial approach failed to detect interaction between INTS13 and other members of the complex (Figure 3a, left panels). Given the structural information for INTS13, the most likely explanation for the inability to detect binding partners is that INTS13 may interact with a domain created by an interface between multiple Integrator subunits. We previously demonstrated this to be the case for the INTS4/9/11 Cleavage Module where INTS4 interaction with INTS9 or INTS11 was only detected with yeast two-hybrid if all three subunits were co-expressed ${ }^{33}$. We therefore decided to focus on whether INTS13 could interact with members of the 
cleavage module and thus repeated the screen with INTS9 and INTS11 expressed simultaneously in trans. Using this approach, we could detect a robust interaction between INTS13 and INTS4 only when INTS9 and INTS11 were co-expressed in the same yeast strain (Figure 3a, right panels and Supplemental Figure 2a). To confirm this interaction, we altered the position of each subunit in the screen and could detect an interaction between INTS9 and INTS13 only when both INTS4 and INTS11 were simultaneously expressed in trans (Supplemental Figure 2b). These data extend on recent structural and biochemical analyses to indicate that INTS13 can, in the absence of INTS10 and INTS14, associate with subunits of the Integrator CM.

The binding interfaces of the Integrator CM have been previously wellcharacterized using both biochemical and structural approaches ${ }^{33,57}$. Based upon these prior analyses, amino acid mutations within each of the three CM subunits are known to disrupt formation of the heterotrimer. Therefore, we repeated our modified two-hybrid assay with each of these mutants tested individually and discovered that any disruption of the INTS4/9/11 CM also results in loss of INTS13 interaction (Figure 3b). Further, in order to confirm the interaction between these four subunits using an independent approach, we co-infected High Five cells with baculoviruses individually expressing Histagged-INTS4, or untagged INTS9, INTS11, and INTS13. The complex was purified from cell lysate using nickel affinity resin and subjected to gel filtration analysis. We observed that all four Integrator subunits remained intact as a complex and could be detected in the same fractions at near stoichiometric levels (Figure $3 c$ ). Collectively, these results indicate that INTS13 interacts with the three components of the Integrator CM only once the module is fully assembled.

We next wanted to determine which region of INTS13 is responsible for this interaction and whether OFD2 mutations may impact it. We tested a series of INTS13 fragments in modified yeast two-hybrid assays to determine whether any of these regions were sufficient to mediate interaction with INTS4/9/11 (Figure 3d schematic). The INTS13 C-terminal region, composed of amino acids 501-706, was as effective as the full-length INTS13 to support growth on selective media (Figure 3d). We further dissected the INTS13 C-terminus through a series of smaller deletion mutants, which ultimately defined that the last 130 residues, amino acids 577-706, was sufficient to bind to the Integrator 
CM (Supplemental Figure 2c). This result is consistent with biochemical approaches recently described where a GST fusion protein containing the C-terminus of INTS13 can pull down subunits of the Integrator $\mathrm{CM}^{56}$. Because both mutations observed in Family 1 (p.K668Nfs ${ }^{*}$ ) and Family 2 (p.S652L) are within the region of INTS13 found to be sufficient to bind INTS4/9/11, we tested whether these mutations could disrupt binding. Indeed, we found that either mutation in the context of the full-length INTS13 protein resulted in a loss of interaction between INTS13 and INTS4/9/11, indicating that this interaction may be specifically disrupted in the four affected patients (Figure 3d).

\section{The INTS13 C-terminus connects an INTS10/13/14 submodule to the Integrator} complex

We next sought to determine if the C-terminal INTS13 mutations have an impact on the assembly of the entire Integrator complex. To do so, we created individual 293T cell lines harboring three different versions of the INTS13 cDNA: wild type (wt), Family 1 mutant (F1), or Family 2 mutant (F2). Each of these lines expresses exogenous INTS13 with an $\mathrm{N}$-terminal FLAG tag under the control of the doxycycline-inducible promoter. After 48 hours of doxycycline treatment, nuclear fractions from each of the cell lines as well as an untransfected naïve line serving as a negative control were extracted. Purified Integrator complexes associated with each INTS13 protein, and the degree of co-associated INT subunits was assessed by LC/MS and Western blot analysis. We observed that both patient-derived INTS13 mutants not only disrupted association with INTS4/9/11, confirming our yeast two-hybrid experiments, but also resulted in reduced binding to nearly all other members of the complex (Figure 4a). The exception to this observation was the binding of INTS14 and to a lesser extent INTS10, which were not as dramatically impacted by the mutations (Figure 4a).

We have shown previously that Integrator constituency and function is highly conserved in Drosophila ${ }^{50}$. Therefore, we wanted to test if patient mutations have the same disruptive effect in the orthologous fly complex. Supporting this approach, the Cterminal region of INTS13 is conserved in the Drosophila IntS13 orthologue (Asunder or dlntS13) and so we introduced the analogous patient mutations into dlntS13 (Supplemental Figure 3a). We then created individual S2 cell lines expressing copper- 
342 inducible FLAG-dIntS13-wild type (wt), Family 1 mutant (dF1), or Family 2 mutant (dF2).

343 We generated nuclear extract from these cell lines as well as a naïve S2 control and 344 purified Drosophila Integrator complexes using anti-FLAG affinity resin. Similar to the 345 human Integrator purifications, we used both LC/MS and Western blot analysis to assess 346 complex integrity. In accordance with the human data, we found that patient mutation 347 'equivalents' within Drosophila IntS13 reduced association with most other Integrator subunits (Figure $4 \mathrm{~b}$ ). We also observed that association of dlntS10 and dlntS14 were only marginally affected by the patient mutations.

These results confirm that OFD2-causing mutations disrupt INTS13 assembly into Integrator, but also suggest that INTS13 mutants do not affect INTS14 and possibly INTS10 binding. Therefore, we wanted to determine whether we could detect an interaction of INTS13 with INTS10 and INTS14 using our modified two-hybrid assay and if INTS13 mutations affected it. We initially expressed INTS14 fused to the Gal4 DNA binding domain and screened for interactions individually with each of the other Integrator subunits fused to the activating domain. This approach did not detect any pairwise interactions (Supplemental Figure 3b, left panel). Therefore, we expressed each Integrator subunit individually in trans with INTS14 fused to the DNA binding domain and INTS13 fused to the activating domain, and we found that the additional presence of INTS10 can allow for an interaction between INTS14 and INTS13, supporting the model that these three subunits form a module as has been recently shown biochemically ${ }^{56}$ (Supplemental Figure 3b, right panel). Finally, we tested whether our patient-derived mutations within INTS13 affect the interaction of INTS10/13/14 and observed that both INTS13 mutants could interact with INTS10/14 comparable to the wild-type INTS13 (Figure 4c). Overall, the results presented here provide a molecular basis for OFD2 where INTS13 mutations disrupt interaction between the INTS10/13/14 submodule and the INTS4/9/11 CM, along with the rest of the Integrator complex (Figure 4d).

Reduced INTS13 expression in RPE cells leads to loss of ciliogenesis and broad disruption of transcriptional regulation of ciliary genes.

371 Results presented thus far reveal that OFD2 patient cells exhibit disrupted ciliogenesis 372 and that these same mutations also disrupt the INTS13 module's interaction with the CM. 
373 We next wanted to determine whether reduced expression of INTS13 in cultured cells

374 was sufficient to inhibit ciliogenesis. We utilized human retinal pigment epithelial (RPE)

375 cells as a model, because these cells exhibit clearly visible primary cilium upon serum

376 starvation. We treated RPE cells with either control siRNA or one of two distinct siRNA

377 targeting INTS13 and discovered that either siRNA could effectively deplete endogenous

378 INTS13 (Figure 5a). To visualize primary cilia, we probed fixed cells using antibodies

379 raised to $A D P-r i b o s y l a t i o n$ factor-like protein $13 B(A R L 13 B)^{59}$, which stains the ciliary

380 axoneme, and $y$-tubulin, which stains the basal body ${ }^{39}$. We observed that the basal body

381 and axoneme components were still present and appropriately juxtaposed in INTS13-

382 depleted cells but were clearly not utilized to form a normal primary cilium (Figure 5b).

383 We found that depletion of INTS13 with either siRNA reduced the number of properly assembled primary cilium by $\sim 50 \%$ relative to control siRNA (Figure $5 \mathrm{c}$ ).

To understand the underlying changes in gene expression that occurred after

386

387

388

389

390

391

392

393

394

395

396

397

398

399

400

401

402

403

INTS13 depletion, we subjected control siRNA-treated and each of the INTS13 siRNAtreated RPE cells to RNA-seq analysis. We conducted RNA-seq using three biological replicates from cells treated with each siRNA and used DESeq to determine differential expression (Supplemental Table 1). Taking advantage of two distinct INTS13 siRNAs, we only considered genes observed to be significantly upregulated/downregulated (>2-fold, $p<0.0001$ ) in both knockdown conditions. Using these filters, we identified 254 genes that were upregulated and 156 genes that were downregulated upon INTS13 knockdown (Figure $5 d$ ). Gene ontology analysis revealed a striking enrichment in genes involved in cilium biogenesis uniquely within the upregulated gene set, suggesting that knockdown of INTS13 leads to de-repression of a collection of genes involved in cilium biogenesis (Figure 5e). We validated several of these gene changes using RT-qPCR including wellcharacterized ciliary genes such as BBOF1, CFAP206, and CFAP53 (Figure 5f). Altogether, these results indicate that depletion of INTS13 in human cells leads to disrupted ciliogenesis that is likely caused by broad transcriptional deregulation of genes involved in primary cilium biogenesis.

\section{Knockdown of ints13 causes developmental defects and ciliary phenotypes in Xenopus.}


404 To examine the role of INTS13 in vivo, we first examined its expression in Xenopus laevis

405

406

407

408

409

410

411

412

413

414

415

416

417

418

419

420

421

422

423

424

425

426

427

428

429

430

431

432

433

434 embryos using whole mount in situ hybridization (WISH). ints13 showed both maternal and zygotic contributions with relatively ubiquitous expression. At late gastrula (stage 12), the expression of ints13 was enriched in ectodermal lineages, with no apparent expression in the endoderm. At neurula (stage 17), its expression was enriched in the neural tube and neural folds (Supplemental Figure 5a). From tadpole stages onwards, ints 13 transcripts were abundantly observed in ciliated tissues and organs including the brain, branchial arches, pronephros, eye and otic vesicles. Remarkably ints 13 was found to follow a punctate pattern of expression in the skin, which coincided with that of multiciliated cells (MCCs). This was validated by whole mount antibody staining, using the a-M antibody highlighting that endogenous INTS13 was enriched in epidermal cells endowed with multicilia (Supplemental Figure 5b).

In order to gain insight into the precise function of ints13 during Xenopus embryonic development, we performed loss-of-function analysis using a morpholino oligonucleotide (MO) targeted against the ints13 start codon (IntS13-MO). This IntS13$\mathrm{MO}$ was previously established to be specific ${ }^{47}$, and we confirmed the translational block efficiency achieved by this MO by demonstrating that ints13 morphants were nearly devoid of endogenous ints13 protein (Figure 6d). The Ints13-MO resulted in developmental delay, a small head, shortened body axis, bent tail, cardiac oedema, polyploidy and early developmental arrest in Xenopus morphants (Figure 6a). In addition to the previously described gastrulation defects, we also observed prominent deficiencies in ciliogenesis and craniofacial development. Based on the enrichment of ints13 in MCCs, from which protrude hundreds of beating cilia ${ }^{60,61}$, we examined the possibility that ints 13 may affect multi-cilia formation and function. In situ hybridization using a specific MCC marker ccdc1962 showed that morphants had significantly less ccdc19 than did control embryos (Figure 6b), which was validated by whole mount antibody staining using the anti-acetylated $\alpha$-tubulin antibody marking cilia protruding from MCCs (Figure 6c). We noted increased distances between puncta and irregular staining in ints13-depleted embryos, suggesting that the epidermis of ints13-depleted tadpoles could have fewer differentiated MCCs and/or that these cells harbor fewer cilia. Scanning electron microscopy (SEM) performed on both ints13 morphants and control embryos revealed 
435 that MCCs were defective, with a significantly reduced number of cilia per cell (Figures

$4366 \mathrm{e}, \mathrm{f})$. While the length of cilia seemed to be normal, inconsistency was observed within and between MCCs, suggestive of incomplete penetrance (Figure 6e). Cilia exhibited abnormalities in morphology, protrusion and direction (Figure 6f). Of note, we were unable to detect overt abnormalities in the ultrastructure of the cilium by transmission electron microscopy (TEM) (Figure 6g). These results prompted us to examine the function of the cilia, which normally generate an anterior-to-posterior directional flow over the tadpole's skin. Using high-resolution imaging, time-lapsed movies revealed that morphants were completely unable to move liquid around their bodies compared to control embryos (Supplemental Movie 1). Moreover, we found that ciliary beating in ints13-depleted embryos was slow, erratic and uncoordinated compared to control embryos (Supplemental Movie 2). These data indicate that INTS13 is necessary for MCC differentiation, as well as cilia formation and beating, thus revealing its critical role in ciliogenesis during development.

\section{Discussion}

Oral facial digital syndromes are an expanding group of genetic conditions characterized by malformations of the face, oral cavity, and digits. These disorders exhibit extensive variability of clinical features with intra-familial and inter-familial variability. Here we describe two homozygous germline mutations in the INTS13 gene in two independent families, with Family 1 fulfilling clinical criteria for OFD. Through molecular and biochemical approaches, we determined that INTS13 associates with INTS10/14 to form a heterotrimer that interacts with the Integrator CM through the C-terminus of INTS13. The two disease-causing mutations both reduce steady-state levels of endogenous INTS13 protein in patient cells and disrupt INTS13 interaction with the CM, likely causing

461 transcriptional defects in a broad collection of ciliary genes. The binding of INTS10/13/14 462 to the Integrator CM is clearly vital, as depletion of INTS13 was sufficient to disrupt 463 ciliogenesis in developing Xenopus embryos. These results underscore both the 464 importance of Integrator assembly during development and reveal the importance of Integrator transcriptional regulation in ciliogenesis. 
INTS13 and INTS14 were not initially described as components of Integrator ${ }^{25}$, but were subsequently discovered through a genome-wide RNAi screen for factors regulating UsnRNA 3' end formation ${ }^{50}$. Since that initial identification, INTS13 has been shown to play a key role in the differentiation of monocytes ${ }^{63}$. During the course of this manuscript preparation, we became aware of another study in which the structure of INTS13 and INTS14 as a heterodimer was solved ${ }^{56}$. Further, using elegant biochemical approaches,

472 Sabath and colleagues determined that INTS13/14 associates into a heterotrimer with 473 INTS10 and the C-terminus of INTS13 binds to the Integrator CM ${ }^{56}$. These structural and 474 biochemical findings are remarkably concordant with results attained here. Interestingly, 475 it was also discovered that the INTS10/13/14 heterotrimer has an overall architecture 476 related to the Ku70-Ku80 DNA repair complex ${ }^{64}$ and is capable of binding DNA or RNA 477 in vitro with the highest affinity for RNA hairpins. Thus, our work here and the structural data in that study establish that Integrator has at least two submodules: the previously defined INTS4/9/11 Cleavage Module containing the RNA endonucleolytic activity and a Nucleic Acid Binding (NAB) module composed of INTS10/13/14. Interaction between these two modules is mediated in part through the C-terminus of INTS13. The structure of the INTS13 C-terminus was not visualized by crystallography, thus obscuring our ability to understand how the germline mutations identified herein disrupt the folding and/or binding of that region ${ }^{56}$. Nevertheless, the collective implication from these two studies is that the Integrator NAB module can bind to RNA hairpins to position the Integrator CM for RNA cleavage and that this process is perturbed in OFD 2/Mohr syndrome patients. phenotypes associated with INTS13 mutation are likely due to a dysfunction in Integratormediated transcriptional regulation. This finding is consistent with previous studies showing that depletion of multiple Integrator subunits can also lead to disrupted ciliogenesis $^{41}$, and that mutations within INTS1 or INTS8 also create ciliopathy-related symptoms $^{42,65}$. Herein, we discovered that depletion of INTS13 causes a $\sim 2$ - to 10-fold upregulation in a broad collection of ciliary genes in RPE cells, which is consistent with recent work demonstrating that depletion of Integrator subunits in Drosophila cells primarily causes an increase in transcription in a subset of genes ${ }^{37,38}$. We posit that an increase in transcription of a broad collection of ciliary genes is sufficient to disrupt 
497 ciliogenesis by potentially overproducing specific components. The concept of dosage 498 imbalance disrupting ciliogenesis has been well documented through reports where gene 499 duplication of centrosome- or cilia-encoding genes can cause ciliopathies ${ }^{66,67,68}$. Even 500 small increases in ciliary gene expression can prove disruptive to ciliogenesis, as 501 evidenced by Down Syndrome patients who present ciliopathy symptoms and, due to its 502 location on chromosome 21 , overproduce Pericentrin a mere $\sim 1.5$-fold ${ }^{69}$. Overall, our 503 results illustrate how sensitive the process of ciliogenesis is to the dosage of components

504

505

506

507

508

509

510

511

512

513

514

515

516

517

518

519

520

521

522

523

524

525

526 and underscores the need for coordinate transcriptional regulation of genes critical to ciliogenesis. It will be interesting in future studies to determine the mechanism by which Integrator coordinately regulates ciliary genes and how disruption of the NAB and Cleavage Modules gives rise to loss of this transcriptional control.

\section{METHODS}

Clinical work. The index cases of the Jordanian family were initially diagnosed at the National Center for Diabetes, Endocrinology \& Genetics in Jordan by Prof. Hanan Hamamy. Clinical assessments were undertaken at different clinics at the University hospital of Jordan. Saliva samples were collected from the whole family members (I.1, I.2, and II.1 to II.6) and skin biopsies were obtained Family 1 (individuals I.2 and II.4). Family 2 was diagnosed at the Imagine Institute in France by Prof. Jeanne Amiel. Blood and skin biopsy were collected from age-matched healthy individuals and patient II:4 from Family 2. Genomic DNA was extracted using (Puregene DNA Purification Kit, Gentra Systems) following the manufacturer's instructions. Nasal epithelial cells were also obtained from (Family 1: I.2, II.4 and II.5) by nasal-brush biopsy (Cytobrush Plus, Medscand, Sweden).

Genomic loci capture. Briefly, custom arrays (Agilent 244K) were designed to target every exonic sequence of the genes present in the five homozygous IBD regions in Family 1 (chr6:167,901,317-170,287,795); (chr8:106,743,988-122,186,235); (chr12:12,129,35028,091,505); (chr13:21,704,404-26,337,017); (chr16:58,296,974-65,631,053). A DNA 
527 library from patient (II:4) was prepared according to the Illumina library generation

528 protocol version 2.3 and hybridized to custom arrays according to the Agilent CGH $244 \mathrm{~K}$ 529 array protocol, and washed, eluted and amplified. The sample was submitted to one 530 channel of Illumina flow cell and sequenced by Illumina Genome Analyzer (GAII) using 531 standard manufacturer's protocol. The image data was processed by the provided GA 532 Pipeline (Firecrest version 1.3.4 and Bustard version 1.3.4) and all sequences were 533 aligned to the human reference genome (UCSC, build 18) by Blat-like Fast Accurate 534 Search Tool (BFAST) ${ }^{70}$. Mismatches were further filtered to identify variants seen ten or more times, in which the variant was called as homozygous and did not overlap with a known dbSNP129 entry mismatch. Nonsynonymous mutations were identified with additional SeqWare tools and the "knownGene" gene model from the UCSC hg18. The open-source SeqWare project that provides a LIMS tool for tracking samples (SeqWare LIMS) and a pipeline for sequence analysis (SeqWare Pipeline) was used throughout this 540 work.

For exome sequencing in Family 2, sequences were aligned to the human genome reference sequence (UCSC Genome Browser, GRCh38 build) by Burrows-Wheeler Aligner. Downstream processing was carried out with the Genome Analysis Toolkit (GATK), SAMtools and Picard Tools. Substitution calls were made with GATK Unified Genotyper, whereas indel calls were made with a GATK IndelGenotyperV2. All calls with a read coverage $\leq 2 X$ and a Phred-scaled single-nucleotide polymorphism (SNP) quality of $\leq 20$ were filtered out. Variants were annotated using an in-house-developed annotation software (PolyWeb) allowing filtering of variants according to relevant genetic models.

Mutation analysis. Positional candidate genes were obtained from GenBank and Ensembl databases. Segregation analysis was done for candidate mutations by Sanger

552 sequencing with the BigDye Terminator cycle sequencing kit (Applied Biosystems) using 553 primers flanking each mutation. The primer sequences for INTS13 (NM_018164) and SACS (NM_014363) mutations screening are given in Supplemental Table 2.

555 Cell culture and transfections. Mouse fibroblast cells and hTERT transformed RPE1 556 cells were cultured in DMEM and Ham's F12/DMEM (Invitrogen), respectively. 557 Lymphocytes cells were grown in cell culture medium (RPMI) (Invitrogen). All cultured 
558 media were supplemented with 10\% Fetal Bovine Serum (FBS) (HyClone), 1X Penicillin

559 / Streptomycin and $2 \mathrm{mM} \mathrm{L-glutamine} \mathrm{under} \mathrm{standard} \mathrm{conditions}\left(37^{\circ} \mathrm{C}, 5 \% \mathrm{CO} 2\right)$. To

560 induce ciliogenesis, cells were grown in serum starvation medium (0.5\% FBS) for 72

561 hours. Transient transfection was done using Lipofectamine 2000 (Life Technologies)

562 according to manufacturer's recommended protocol.

HEK 293T (ATCC, Manassas VA, CRL-3216) cells were grown at $37^{\circ} \mathrm{C}$ with $5 \%$

564

565

566

567

568

569

570

571

572

573

574

575

576

577

578

579

580

581

582

583

584

585

586

587 $\mathrm{CO}_{2}$ in DMEM (Gibco, Waltham MA, \#11965-092), supplemented with $10 \%(\mathrm{v} / \mathrm{v}) \mathrm{FBS}$ and 1\% (v/v) penicillin-streptomycin (Gibco, \#15070-063). RPE cells (ATCC, ARPE-19, CRL2302, lot $\# 70004873$ ) were grown at $37^{\circ} \mathrm{C}$ with $5 \% \mathrm{CO}_{2}$ in DMEM/F-12 50/50 (Corning, Manassas VA, 10-092-CV), supplemented with $10 \%(\mathrm{v} / \mathrm{v})$ FBS and $1 \%(\mathrm{v} / \mathrm{v})$ penicillinstreptomycin. Drosophila S2 cells were grown at $27^{\circ} \mathrm{C}$ in Sf-900 II SFM (Gibco, \#10902088), supplemented with 1\% (v/v) Antibiotic-Antimycotic (Gibco, 15240-062).

293T cells were transfected using CRISPR/Cas9 to make inducible stable lines. 300,000 cells per well were seeded in a 6-well plate. The following day, $2 \mu \mathrm{l}$ of Lipofectamine 2000 (Invitrogen, Carlsbad CA, 11668-019) was combined with $100 \mu \mathrm{l}$ of Opti-MEM I (Gibco, 31985-070) per well and incubated at room temperature for 7 minutes. $100 \mu \mathrm{l}$ of this solution was added to $500 \mathrm{ng}$ of the Cas9 AAVS1 sgRNA plasmid and $500 \mathrm{ng}$ of a pTet-3xFLAG-INTS13 plasmid per well and incubated at room temperature for 20 minutes. The transfection solution was then added to wells containing $1 \mathrm{ml}$ of media. Each insert gene was done in duplicate wells. The next day, duplicate wells were combined, and cells were expanded to a $10-\mathrm{cm}$ dish. 48 hours after transfection, stable cell selection began with $1.5 \mu \mathrm{g} / \mathrm{ml}$ puromycin. Once stable lines were created, cells were maintained at $1 \mu \mathrm{g} / \mathrm{ml}$ puromycin. Human INTS13 cDNA was mutated by sitedirected PCR to create the genes for Family 1 and Family 2. All three versions of the INTS13 gene, including wild type, were cloned individually into a plasmid containing the tetracycline-inducible Tet-On $3 G$ promotor with a $3 x-F L A G$ tag for insertion into the AAVS1 locus. This was accomplished by co-transfection with the Cas9 plasmid containing the AAVS1 guide RNA (ACCCCACAGTGGGGCCACTA). Protein expression for nuclear extract was induced for 48 hours with $0.4 \mu \mathrm{g} / \mathrm{ml}$ doxycycline for INTS13 wild type and Family 1 and $12 \mu \mathrm{g} / \mathrm{ml}$ for Family 2 in 30 15-cm diameter dishes per line. 
Stable lines were also made with S2 cells. Drosophila INTS13 cDNA was mutated using site-directed PCR to make orthologous versions of the Family 1 and Family 2 mutations indicated in Supplemental Fig 3. The Family I cDNA also had an early stop codon added to recapitulate the truncation seen in the human gene. These modified genes, and wild type INTS13, were cloned into a pMT-3xFLAG-puro plasmid ${ }^{37}$ following the metallothionein promotor and $3 \times$-FLAG tag. $2 \times 10^{6}$ cells were plated in Schneider's media supplemented with $10 \%$ FBS in a 6 -well plate overnight. $2 \mu \mathrm{g}$ of plasmid was transfected using Fugene HD (Promega, Madison WI, \#E2311). Plasmid DNA was mixed with $8 \mu \mathrm{L}$ Fugene and $100 \mu \mathrm{L}$ media and incubated at room temperature for 15 minutes before being added to cells. After 24 hours, $2.5 \mu \mathrm{g} / \mathrm{mL}$ puromycin was added to the media to select and maintain the cell population. Cells were transitioned to SFX media without serum for large scale growth. Protein expression for nuclear extract was induced by adding $500 \mathrm{mM}$ copper sulfate for 48 hours to 1 liter of each cell line grown to approximately $1 \times 10^{7}$ cells $/ \mathrm{mL}$.

Immunofluorescence staining. For patient cells: cells were seeded on $12 \mathrm{~mm}$ cover slips. Cells were grown to around $80 \%$ confluency and then incubated with $0.5 \%$ serum media for 72 hours to induce cilia formation, washed and kept at $4^{\circ} \mathrm{C}$ until use. Nasal epithelial cells were suspended in RPMI without supplements to avoid artefacts caused by serum. The cytobrush was moved gently up and down for $5 \mathrm{~min}$ to detach ciliary cells from the brush. Samples were then spread onto non-coated glass slides, air dried, and stored at $-80^{\circ} \mathrm{C}$ until use. (See our protocol for immunofluorescence staining, URL)

For RNAi-treated RPE cells (Figure 5b): After RNAi treatment (described in the RNA interference section) and serum starvation of cells grown on cover slips, cells were washed with DPBS and each cover slip was placed in an individual well of a 24-well plate. Cells were fixed to the cover slips by incubating in ice cold methanol for 7-8 minutes and washed thoroughly with PBS. Then samples were blocked for $30 \mathrm{~min}$ (all incubation steps done at $37^{\circ} \mathrm{C}$ with humidity). The blocking buffer used was $1 \%$ bovine serum albumin (Sigma-Aldrich, St. Louis MO, A7906-50G) (w/v) in PBS $+0.1 \%$ Tween 20 (Fisher Scientific, Fair Lawn NJ, BP337-500). Probing was performed for 2 hours with the following primary antibodies at 1:1000 in blocking buffer: rabbit anti-gamma tubulin 
619 (Proteintech, Rosemont IL, 15176-1-AP) and mouse anti-ARL13B (Proteintech, 66739-1-

$620 \mathrm{lg}$ ), followed by three washes with PBS for 5 minutes each. Secondary antibody probing 621 was for 1 hour in the dark with the following antibodies at 1:1000 in blocking buffer: goat 622 anti-rabbit Alexa Fluor 594 (Life Technologies, Carlsbad CA, A11037) and goat anti623 mouse Alexa Fluor 488 (Life Technologies, A11029), followed by three more washes with 624 PBS. Cover slips were mounted on slides using Fluoroshield Mounting Medium with DAPI 625 (Abcam, Cambridge, UK, ab104139). Confocal imaging was performed on a Leica TCS626 SP8 3X system (Leica Microsystems, Wetzlar Germany) equipped with a Leica HC PL 627 APO CS2 63×/1.4 oil immersion objective. A tunable (470-670 nm) pulsed white light laser 628 (Leica WLL) and a UV $405 \mathrm{~nm}$ diode laser was used for excitation. Images were 629 processed with the image analysis software, Imaris Pro 9.3.0.

Reverse transcription (RT-PCR) and Quantitative PCR. Figure 2: Total RNA was isolated from cells or embryos using Trizol (Ambion by Life Technologies, Carlsbad CA, 15596018) followed by extraction using the RNeasy Mini Kit (Qiagen, Hilden Germany). RNA $(1 \mu \mathrm{g})$ was reverse transcribed using Iscript ${ }^{\text {TM }}$ cDNA Synthesis Kit (Bio-transcript lead) and the mis-splicing of the transcript was assessed using pair of primers designed in the exons upstream and downstream of the morpholino binding site. Quantitative realtime PCR was performed with power SYBR green master mix (Applied Biosystems) using 638 a 7300 real-time polymerase chain reaction machine (Applied Biosystems) and 639 normalized to the housekeeping gene $\beta$-actin. Primer sequences for RT-PCR and quantitative real-time PCR are listed in the Supplemental Table 2.

Figure 5: Total RNA was isolated using Trizol for phenol-chloroform extraction. 642 cDNA was reverse transcribed using Superscript III Reverse Transcriptase (Thermo 643 Scientific, Rockford IL, \#18080085) according to the manufacturer's instructions. Random 644 hexamers were used for cDNA synthesis primers and qRT-PCR was then carried out in 645 triplicate using Bio-Rad iTaq Universal SYBR Green Supermix (Bio-Rad, Hercules CA, $646 \quad \# 1725120)$ and measured with the CFX Connect Real-Time System (Bio-Rad). Data was 647 analyzed using the $\Delta \Delta \mathrm{Ct}$ method to calculate fold change, with $7 S K$ as the control gene. 
648

649

650

651

652

653

654

655

656

657

658

659

660

661

662

663

664

665

666

667

668

669

670

671

672

673

674

675

676

677

Morpholino, RNA injection and embryological methods. The ints13 morpholino (MO) purchased from Gene Tools were resuspended in sterile water to a concentration of 1 $\mathrm{mM}$ according to the manufacturer's instructions. RNA was then extracted and converted to cDNA. To target non-neural ectoderm, 1:4 of diluted ints13-MO was injected at the twoto four- cell stage. Morpholino sequences are given in Supplemental Table 2. Protocols for Xenopus fertilization, MO-injections, antibody staining, whole-mount in situ hybridization and immunofluorescence staining are at our protocol website (https://sites.google.com/a/reversade.com/www/protocols/). All experiments with Xenopus embryos were approved by the Singapore National Advisory on Laboratory Animal Research. A stereomicroscope equipped with an ICD digital camera (Leica M205 FA) was used to capture images of embryos in successive focal planes. Images were then combined into one picture with Photoshop CS6 (Adobe).

Western Blot. For Figures 2 and 6: Cells or embryos were lysed in RIPA buffer supplemented with a cocktail of protease inhibitor (Roche). Total protein was resolved on SDS polyacrylamide gels (Bio-Rad) with DTT, followed by transfer on polyvinylidene difluoride (PVDF) membranes. The following antibodies were used for HRP-mediated chemiluminescent: polyclonal Rabbit antibody to INTS13 (C \& M), monoclonal Mouse antibody to actin (Chemicon; Mab1501R), $\beta$-tubulin (E7, Developmental Studies Hybridoma Bank, University of lowa, lowa City, IA), dynein heavy chain ( $\mathrm{P} 1 \mathrm{H} 4$, gift from T. Hays, University of Minnesota, Minneapolis, MN) and anti-HA antibody (Sigma, H 6533 (clone HA-7).

For Figures 4 and 5: Protein was extracted directly from RPE cells treated with siRNA by adding 2 X SDS loading buffer (120mM Tris pH6.8, 4\% SDS, 200mM DTT, $20 \%$ Glycerol, and $0.02 \%$ Bromophenol blue) to cells while on the plate. Samples were then incubated at room temperature while on the plate with periodic swirling prior to a 10minute boiling at $95^{\circ} \mathrm{C}$ and a short sonication. Denatured protein samples were then resolved on a $10 \%$ polyacrylamide gel and transferred to a PVDF membrane (Thermo Scientific, 88518). Blots were probed as previously described ${ }^{37}$. Samples from nuclear extract immunoprecipitation are described in the anti-FLAG affinity purification. 
678 Electron microscopy (EM). Embryos at the desired stages were fixed in 3\% PBS679 glutaraldehyde, $\mathrm{pH} 7.4$, for 4 hours with agitation. After 3 washes in fresh phosphate 680 buffer $(\mathrm{NaH} 2 \mathrm{PO} 4,2 \mathrm{H} 2 \mathrm{O} / \mathrm{Na} 2 \mathrm{HPO} 4,12 \mathrm{H} 2 \mathrm{O})$ for 5 minutes each with vigorous agitation, 681 embryos were rinsed 3 times / 5 minutes in distilled water, dehydrated in ethanol with 682 vigorous agitation, dried in Leica EM CPD030 and sputter coated in gold coating, 7nm 683 thick layer using Leica SCD050. Embryos were viewed using a scanning electron 684 microscopy (FESEM, JEOL, JSM-6701F). For Transmission electron microscopy (TEM) 685 dehydrated embryos were embedded in Epon 812, prepared sections (50-60 nm) and 686 contrasted with $4 \%$ uranyl acetate in $50 \%$ ethanol and $2.6 \%$ lead nitrate in $1 \mathrm{M} \mathrm{NaOH}$. 687 Embryos were scanned using a Hitachi 7000 electron microscope (Hitachi, Tokyo, 688 Japan). EM scanning was performed at electron microscopy facility (Institute of Molecular 689 and Cell Biology (IMCB), A*STAR, Singapore).

Plasmid construction and Yeast two-hybrid analysis. Yeast two-hybrid analysis was carried out in PJ69-4 $\alpha$ and PJ49-4a strains as described ${ }^{33}$. In brief, human INTS1 through 692 INTS14, and INTS13 variations, were cloned into pGAD, pOBD, pTEF, or pCEVG1Ura3/TEFdual vectors using conventional cloning. pOBD plasmids were then transformed into PJ69-4 $\alpha$ yeast and were selected on tryptophan-dropout medium (Clontech, Mountain View CA, \#630413); pGAD plasmids were transformed into PJ49-4a yeast and were selected on leucine-dropout medium (Clontech, \#630414). pTEF or pTEFdual plasmids were included to express proteins in trans and were transformed with pOBD plasmids and selected on media lacking tryptophan and uracil (Sunrise Science Products, San Diego CA, 1316-030). Yeast strains were then mated and subsequently selected on medium lacking tryptophan, leucine, and uracil (Sunrise Science Products, 1328-030). Interactions were tested through serial dilution (one to five) of diploid yeast followed by plating on medium lacking tryptophan, leucine, and uracil or on medium lacking tryptophan, leucine, uracil, and histidine (Sunrise Science Products, 1334-030) 704 that also was supplemented with $1 \mathrm{mM}$ 3-amino-1,2,4-triazole (MP Biomedicals, Irvine 705 CA, 4061-722). 
708

709

710

711

712

713

714

715

716

717

718

719

720

721

722

723

724

725

726

727

728

729

730

731

732

733

734

735

736

737

Multibac technology (Geneva Biotech) ${ }^{71}$. INTS4 carried an N-terminal hexa-histidine tag while the other three proteins were untagged. High Five cells were grown in ESF 921 medium (Expression Systems) by shaking at $120 \mathrm{rpm}$ at $27^{\circ} \mathrm{C}$ until the density reached $1.5-2 \times 10^{6}$ cells $\mathrm{mL}^{-1}$. Cells were infected by $\mathrm{P} 1$ viruses and harvested after $48 \mathrm{~h}$. The cells were lysed by sonication in a buffer containing $20 \mathrm{mM}$ Tris (pH 8.0), $200 \mathrm{mM} \mathrm{NaCl}$, $5 \%(\mathrm{v} / \mathrm{v})$ glycerol and $0.1 \mathrm{mM}$ PMSF. The supernatant of cell lysates was incubated with nickel beads (Qiagen) and the eluate was then subjected to gel filtration chromatography (Superdex 200, GE Healthcare), in a running buffer of $20 \mathrm{mM}$ Tris (pH 8.0), $250 \mathrm{mM} \mathrm{NaCl}$, and $5 \mathrm{mM}$ DTT.

Nuclear extract. Cells were collected and washed in cold PBS. 293T cells were detached from dishes while $S 2$ cells were grown in suspension and pelleted by centrifugation. Cells were then resuspended in five times the cell pellet volume of Buffer A (10mM Tris pH8, $1.5 \mathrm{mM} \mathrm{MgCl}_{2}, 10 \mathrm{mM} \mathrm{KCl}, 0.5 \mathrm{mM}$ DTT, and 0.2mM PMSF). Resuspended cells were allowed to swell during a 15 -minute rotation at $4^{\circ} \mathrm{C}$. After pelleting down at $1,000 \mathrm{~g}$ for 10 minutes, two volumes of the original cell pellet of Buffer A was added and cells were homogenized with a dounce pestle B for 20 strokes on ice. Nuclear and cytosolic fractions were then separated by centrifugation at $2,000 \mathrm{~g}$ for 10 minutes. To attain a nuclear fraction, the pellet was washed once with Buffer A before resuspending in an equal amount of the original cell pellet volume of Buffer C (20 mM Tris pH8, $420 \mathrm{mM} \mathrm{NaCl}, 1.5$ $\mathrm{mM} \mathrm{MgCl}_{2}, 25 \%$ glycerol, $0.2 \mathrm{mM}$ EDTA, $0.5 \mathrm{mM} \mathrm{PMSF}$, and $0.5 \mathrm{mM}$ DTT). The sample was then homogenized with a dounce pestle B for 20 strokes on ice and rotated for 30 minutes at $4^{\circ} \mathrm{C}$ before centrifuging at $15,000 \mathrm{~g}$ for 30 minutes at $4^{\circ} \mathrm{C}$. Finally, supernatants were collected and subjected to dialysis in Buffer D (20 mM HEPES, $100 \mathrm{mM} \mathrm{KCl}, 0.2$ $\mathrm{mM}$ EDTA, $0.5 \mathrm{mM} \mathrm{DTT}$, and $20 \%$ glycerol) overnight at $4{ }^{\circ} \mathrm{C}$. Prior to any downstream applications, nuclear extracts were centrifuged again at $15,000 \mathrm{~g}$ for 3 minutes at $4^{\circ} \mathrm{C}$ to remove any precipitate.

Anti-FLAG affinity purification. To purify FLAG-tagged Integrator complexes for mass spectrometry, generally between 8 and $10 \mathrm{mg}$ of nuclear extract (approximately $1.9 \mathrm{~mL}$ of extract depending on the concentration) was mixed with $100 \mu \mathrm{L}$ anti-Flag M2 affinity agarose slurry (Sigma-Aldrich, \#A2220) washed with $0.1 \mathrm{M}$ glycine then equilibrated in 
738

739

740

741

742

743

744

745

746

747

748

749

750

751

752

753

754

755

756

757

758

759

760

761

762

763

764

765

766

767

768

binding buffer (20 mM HEPES pH7.4, $150 \mathrm{mM} \mathrm{KCl,} \mathrm{10 \%} \mathrm{Glycerol,} \mathrm{0.1 \%} \mathrm{NP-40).} \mathrm{This}$ mixture was rotated for four hours at $4^{\circ} \mathrm{C}$. Following the four-hour incubation/rotation, five sequential washes were carried out in binding buffer with a 10 -minute rotation at $4^{\circ} \mathrm{C}$ followed by a $1,000 \mathrm{~g}$ centrifugation at $4^{\circ} \mathrm{C}$. After a final wash with $20 \mathrm{mM}$ HEPES buffer, the supernatant was removed using a pipette and the beads were kept cold and submitted to the mass spectrometry core where the protein complexes were eluted by digestion (described below). For immunoprecipitation samples intended for Western blot, a similar protocol was used. $25 \mu \mathrm{L}$ of bead slurry and $200 \mu \mathrm{L}$ of extract sample were rotated for two hours at $4^{\circ} \mathrm{C}$. After the fifth wash with binding buffer, protein complexes were eluted from the anti-FLAG resin by adding $50 \mu \mathrm{L}$ of $2 \mathrm{X}$ SDS loading buffer and boiled at $95^{\circ} \mathrm{C}$ for five minutes. For Western blots, input samples were generated by adding equal volume of $2 X$ SDS loading buffer to nuclear extract and 1/10 of the immunoprecipitation was loaded as estimated by protein mass.

Mass spectrometry sample digestion. The samples were prepared in a similar manner as described previously ${ }^{72}$. Briefly, the agarose bead-bound proteins were washed several times with $50 \mathrm{mM}$ Triethylammonium bicarbonate (TEAB) pH 7.1, before being solubilized with $40 \mu \mathrm{L}$ of $5 \%$ SDS, $50 \mathrm{mM}$ TEAB, $\mathrm{pH} 7.55$ followed by a room temperature incubation for 30 minutes. The supernatant containing the proteins of interest was then transferred to a new tube, reduced by making the solution $10 \mathrm{mM}$ Tris(2-carboxyethyl)phosphine (TCEP) (Thermo, \#77720), and further incubated at $65^{\circ} \mathrm{C}$ for 10 minutes. The sample was then cooled to room temperature and $1 \mu \mathrm{L}$ of $1 \mathrm{M}$ iodoacetamide acid was added and allowed to react for 20 minutes in the dark. Then, $5 \mu \mathrm{L}$ of $12 \%$ phosphoric acid was added to the $50 \mu \mathrm{L}$ protein solution followed by $350 \mu \mathrm{L}$ of binding buffer ( $90 \%$ Methanol, $100 \mathrm{mM}$ TEAB final; $\mathrm{pH}$ 7.1). The resulting solution was administered to an S-Trap spin column (Protifi, Farmingdale NY) and passed through the column using a bench top centrifuge (30 second spin at $4,000 \mathrm{~g}$ ). The spin column was then washed three times with $400 \mu \mathrm{L}$ of binding buffer and centrifuged (1200 rpm, $1 \mathrm{~min}$ ). Trypsin (Promega, \#V5280) was then added to the protein mixture in a ratio of 1:25 in $50 \mathrm{mM} \mathrm{TEAB}, \mathrm{pH}=8$, and incubated at $37^{\circ} \mathrm{C}$ for 4 hours. Peptides were eluted with $80 \mu \mathrm{L}$ of $50 \mathrm{mM}$ TEAB, followed by $80 \mu \mathrm{L}$ of $0.2 \%$ formic acid, and finally $80 \mu \mathrm{L}$ of $50 \%$ acetonitrile, $0.2 \%$ formic acid. The combined 
769

770

771

772

773

774

775

776

777

778

779

780

781

782

783

784

785

786

787

788

789

790

791

792

793

794

795

796

797

798

799

peptide solution was then dried in a speed vacuum (room temperature, 1.5 hours) and resuspended in $2 \%$ acetonitrile, $0.1 \%$ formic acid, $97.9 \%$ water and aliquoted into an autosampler vial.

NanoLC MS/MS Analysis. Peptide mixtures were analyzed by nanoflow liquid chromatography-tandem mass spectrometry (nanoLC-MS/MS) using a nano-LC chromatography system (UltiMate 3000 RSLCnano, Dionex, Thermo Fisher Scientific, San Jose, CA). The nano-LC-MS/MS system was coupled on-line to a Thermo Orbitrap Fusion mass spectrometer (Thermo Fisher Scientific, San Jose, CA) through a nanospray ion source (Thermo Scientific). A trap and elute method was used to desalt and concentrate the sample, while preserving the analytical column. The trap column (Thermo Scientific) was a C18 PepMap100 (300 $\mu \mathrm{m} \times 5 \mathrm{~mm}, 5 \mu \mathrm{m}$ particle size) while the analytical column was an Acclaim PepMap $100(75 \mu \mathrm{m} \times 25 \mathrm{~cm}$ ) (Thermo Scientific). After equilibrating the column in $98 \%$ solvent $A(0.1 \%$ formic acid in water) and $2 \%$ solvent $B$ $(0.1 \%$ formic acid in acetonitrile $(A C N))$, the samples $(2 \mu \mathrm{L}$ in solvent $A)$ were injected onto the trap column and subsequently eluted $(400 \mathrm{~nL} / \mathrm{min})$ by gradient elution onto the C18 column as follows: isocratic at $2 \% \mathrm{~B}, 0-5 \mathrm{~min} ; 2 \%$ to $32 \% \mathrm{~B}, 5-39 \mathrm{~min} ; 32 \%$ to $70 \%$ $\mathrm{B}, 39-49 \mathrm{~min} ; 70 \%$ to $90 \% \mathrm{~B}, 49-50 \mathrm{~min}$; isocratic at $90 \% \mathrm{~B}, 50-54 \mathrm{~min} ; 90 \%$ to $2 \%, 54-$ $55 \mathrm{~min}$; and isocratic at $2 \% \mathrm{~B}$, until the 65 minute mark.

All LC-MS/MS data were acquired using XCalibur, version 2.1.0 (Thermo Fisher Scientific) in positive ion mode using a top speed data-dependent acquisition (DDA) method with a 3 second cycle time. The survey scans ( $\mathrm{m} / \mathrm{z} 350-1500)$ were acquired in the Orbitrap at 120,000 resolution (at $\mathrm{m} / \mathrm{z}=400$ ) in profile mode, with a maximum injection time of $100 \mathrm{~m}$ s and an AGC target of 400,000 ions. The S-lens RF level was set to 60 . Isolation was performed in the quadrupole with a $1.6 \mathrm{Da}$ isolation window, and CID MS/MS acquisition was performed in profile mode using rapid scan rate with detection in the ion-trap using the following settings: parent threshold $=5,000$; collision energy $=32 \%$; maximum injection time $56 \mathrm{msec}$; AGC target 500,000 ions. Monoisotopic precursor selection (MIPS) and charge state filtering were on, with charge states 2-6 included. Dynamic exclusion was used to remove selected precursor ions, with a $+/-10 \mathrm{ppm}$ mass tolerance, for 15 seconds after acquisition of one MS/MS spectrum. 
800 Database Searching. Tandem mass spectra were extracted and charge state 801 deconvoluted using Proteome Discoverer (Thermo Fisher, version 2.2.0388). Deisotoping

802

803

804

805

806

807

808

809

810

811

812

813

814

815

816

817

818

819

820

821

822

823

824

825

826

827

828

829 was not performed. All MS/MS spectra were searched against the appropriate database, either Uniprot Drosophila database (version 04-04-2018) or Uniprot Human database (reviewed 06272018), using Sequest. Searches were performed with a parent ion tolerance of $5 \mathrm{ppm}$ and a fragment ion tolerance of $0.60 \mathrm{Da}$. Trypsin was specified as the enzyme, allowing for two missed cleavages. Fixed modification of carbamidomethyl (C) and variable modifications of oxidation (M) and deamidation were specified in Sequest. Heat maps in Figure 4 were made using Morpheus from the Broad Institute, https://software.broadinstitute.org/morpheus.

RNA interference: siRNA used were Universal Negative Control \#2 (Sigma Aldrich, \#SIC002), INTS13-1 (Sigma Aldrich, SASI_Hs01_00134940/ASUN), and INTS13-2 (Sigma Aldrich, SASI_Hs01_00134941/ASUN). RPE cells were seeded at 50,000 cells/well in a 24-well plate on Day 1. On Day 2, $3 \mu \mathrm{L}$ of $20 \mu \mathrm{M}$ siRNA was added to 50 $\mu \mathrm{L}$ of Opti-MEM I per well and incubated at room temperature for 5 minutes. $2 \mu \mathrm{L}$ of Lipofectamine RNAiMax (Invitrogen, 13778-150) was also added to $50 \mu \mathrm{L}$ of Opti-MEM per well and incubated at room temperature for 5 minutes. The siRNA and RNAi Max solutions were combined and incubated at room temperature for 20 minutes, then added to the cells. Cells were expanded to a 6-well plate on Day 3. On Day 4, cells were washed gently with DPBS and $900 \mu \mathrm{L}$ of DMEM/F-12 media was added to each well. The siRNA treatment from Day 2 was repeated. Cells were further incubated and then harvested on Day 6. For RNA extraction, cells were harvested with $500 \mu \mathrm{L}$ of Trizol. For protein extraction, cells were harvested with SDS loading buffer detailed previously.

A modified version of the above protocol was followed for cells intended for immunofluorescence staining of primary cilia: RPE cells were seeded onto three cover slips in each well of the 6-well plate on Day 3. On Day 4, cells were washed twice with DPBS and grown in serum free (0\% FBS) DMEM/F-12 + 1\% ( $/ \mathrm{v})$ penicillin-streptomycin for 48 hours before fixation. 
830

831

832

833

834

835

836

837

838

839

840

841

842

843

844

845

846

847

848

849

850

851

852

853

854

855

Library preparation and RNA sequencing: RNAi-treated RPE cells were harvested in $500 \mu \mathrm{L}$ of Trizol, and total RNA was purified by phenol-chloroform extraction. $1 \mu \mathrm{g}$ total RNA per sample was used for sequencing library preparation. The NEBNext Poly(A) mRNA magnetic isolation kit (New England Biolabs, Ipswich MA, E7490) was used to purify mRNA from the total RNA. The Click-Seq protocol was used following this step ${ }^{73}$. RT-PCR to make cDNA was done with 1:35 azido-NTP:dNTP for random strand termination by SuperScript III reverse transcriptase. Following Click-Seq preparations, libraries were PCR amplified for 17 cycles and products from 400-600 bp were collected for sequencing. Samples were sequenced on a NextSeq500 (Illumina) using a single read $75 \mathrm{bp}$ run. Sequencing adapters removed and reads with a Qscore $<30$ were discarded using fastp (ver 0.14.1). Reads were aligned using Hisat2 (ver 2.1.0) to the hg38 genome using default parameters. Differential expression changes were determined using DESeq2 (ver 1.23.10) and assigned using the comprehensive gene annotation in GENCODE32 and tests for significance were using the nbinomialWald test. Alignment information and lowest correlation between samples in each data set is given in table below.

\begin{tabular}{|l|l|l|l|l|}
\hline Sample & Total Reads & $\begin{array}{l}\text { Mappable } \\
\text { Fragments } \\
\text { (Percent of total) }\end{array}$ & $\begin{array}{l}\text { Agreement } \\
\text { between } \\
\text { replicates } \\
\text { (Spearman's rho) }\end{array}$ & $\begin{array}{l}\text { Normalization } \\
\text { Factor }\end{array}$ \\
\hline Control & $131,005,450$ & $94.33 \%$ & $>0.99$ & 1.142296033 \\
\hline INTS13-1 & $149,803,846$ & $94.27 \%$ & $>0.99$ & 0.999334293 \\
\hline INTS13-2 & $168,253,066$ & $94.13 \%$ & $>0.99$ & 0.889755677 \\
\hline
\end{tabular}

\section{ACKNOWLEDGMENTS}

We thank all families for partaking in this study. The authors would also like to thank members of their team for technical assistance and fruitful discussions. This work was supported by a grant from the French Ministry of Health to J.A., Strategic Positioning Fund on Genetic Orphan Diseases from the Biomedical Research Council, A*STAR, Singapore to B.R., and by the UCLA California Center for Rare Diseases to H.L., S.F.N. B.R. is a fellow of the Branco Weiss Foundation, an $A^{*}$ STAR Investigator and Young EMBO Investigator. This work was supported by National Institutes of Health grant R01- 
856

857

858

859

860

861

862

863

864

865

866

867

868

869

870

871

872

873

874

875

876

877

878

879

880

881

882

876

GM134539 (E.J.W.), and the Welch Foundation grant H-1889 to The University of Texas Medical Branch at Galveston (E.J.W.). The UTMB Mass Spectrometry Facility is supported in part by The Cancer Prevention Research Institute of Texas (CPRIT) grant number RP190682 (W.K.R). M.K.S. was supported by CPRIT grant number P71709-B01.

\section{COMPETING FINANCIAL INTERESTS}

The authors declare no competing financial interests

\section{AUTHOR CONTRIBUTIONS}

L.G.M. and M.S. contributed equally to this work. B.R. initiated this project with H.H. who clinically ascertained Family 1 with the support of B.A.R., O.A. and M.E.K. Family 2 was ascertained by J.A. and L.C. L.G.M. conducted RPE analysis, biochemical purifications, and yeast two-hybrid analysis. M.S. performed all experiments on patients' primary cells and in Xenopus with the help of N.E.B. N.D.E. prepared samples for and analyzed RNAseq. K-L.H. performed Western blot analysis. N.P. built the pTEFdual vector. H.L., S.F.N., and B.M. conducted linkage/sequence analysis. Y.X. and L.T. conducted recombinant protein production and gel filtration. M.K.S. and L.J.K. imaged and analyzed PC immunofluorescence. W.K.R. prepared proteomic samples and analyzed LC/MS data. L.G.M., M.S., B.R., and E.J.W. wrote the manuscript. All authors critically reviewed the manuscript.

\section{WEB RESOURCES}

The URLs for data presented herein are as follows:

BLAST, http://blast.ncbi.nlm.nih.gov/Blast.cgi

dbSNP146, http://www.ncbi.nlm.nih.gov/snp

1000 Genomes, http://www.1000genomes.org/

Online Mendelian Inheritance in Man (OMIM), http://www/omim.org/ 
883

884

885

886

887

888

889

890

891

892

893

894

895

896

897

898

899

900

901

902

903

904

905

906

907

908

ExAC, http://exac.broadinstitute.org/

ANNOVAR, http://www.openbioinformatics.org/annovar/

PolyPhen-2, http://genetics.bwh.harvard.edu/pph2/

Human Splicing Finder, http://www.umd.be/HSF3/HSF.html

ClinVar, ftp://ftp.ncbi.nlm.nih.gov/pub/clinvar/vcf GRCh37/

Exome Sequencing Project, http://evs.gs.washington.edu/EVS/

FIGURE LEGENDS

Fig 1. Identification of two families segregating recessive mutations in INTS13. a Pedigree of Family 1 (top): affected individuals (II.4 and II.5) were born to first-cousin parents (I.1 and I.2) with three unaffected children (II.1-II.3). Double lines indicate a consanguineous marriage. Pedigree of Family 2 (bottom): affected individuals (II.2 and II.4) were born to parents with two unaffected children (II.1 and II.3). b Homozygosity mapping of Family 1 delineated five candidate loci totaling $46 \mathrm{cM}$ on chromosomes 6,8 , 12, 13, and 16. c Schematic representation of human INTS13 located on chromosome 12 (ch12p13.2-p11.22) consisting of 17 exons. Locus capture followed by massive parallel sequencing identified a homozygous frameshift mutation as a disease-causing mutation in Family 1. The c.2004delA (p.K668Nfs*9) mutation is caused by a single base pair deletion in exon 16 (marked in brown), resulting in a frame shift and premature termination codon (PTC) which alters nine amino acids (marked in red) and deletes the last 31. In Family 2, the c.1955C > T (S652L) mutation is caused by a single base pair substitution in exon 16 (marked in green). d Amino acid alignment of the C-terminus of the INTS13 protein shows that both mutations occur at highly conserved residues. Red and yellow shading indicates regions of conservation. 
Fig 2. Characterization of Loss-of-function INTS13 mutations in patient cells. a RTqPCR shows that endogenous INTS13 transcript levels were significantly reduced relative to control cells in primary dermal fibroblasts of affected individual II.4 from Family 1. Data are mean $+/-S D, n=3$. Statistical significance was calculated using a two-tailed unpaired t-test. b Primary dermal fibroblasts from individual II.4 from Family 1 were treated with $100 \mathrm{~g} / \mathrm{ml}$ cycloheximide (CHX). Following treatment, RT-qPCR was done which shows that the INTS13 mRNA level was increased in treated cells. Data are mean +/- SEM, $\mathrm{n}=$ 3. Statistical significance was calculated using a one-tailed Student's t-test. c Western blot analysis of primary dermal fibroblasts of affected individual II.4 from Family 1 using two polyclonal antibodies designed against distinct regions of INTS13. No signal was detected in patient cells with the antibody directed against the C-terminus of INTS13 (C). A truncated protein was detected in patient cells by the antibody directed against the center of the protein (M). Actin was used as a loading control. d RT-qPCR shows that INTS13 transcript levels were significantly reduced in primary dermal fibroblast cells of affected individual II.4 from Family 2 relative to control cells. Data are mean +/- SD, $n=$ 3. Statistical significance was calculated using a two-tailed unpaired t-test. e Western blot analysis of primary dermal fibroblasts of affected individual II.4 from Family 2 shows reduced levels of INTS13 protein compared to control cells. $\mathbf{f}$ Western blot analysis of primary dermal fibroblasts from individual II.4 from Family 2 treated with MG132. Treated cells show a level of INTS13 protein comparable to control cells. ${ }^{*}$ indicates a non-specific signal. g Multiciliated airway cells were obtained from nasal biopsies of three members of Family 1: the unaffected carrier mother (Con.) and the two affected children (II.4 and II.5). Probing for acetylated a-tubulin shows significantly shorter, less dense, and disorganized cilia in the affected individuals compared to the mother's cells. $\mathbf{h}$ Bar graph representing the average length of nasal cilia. Data are mean $+/-$ SEM, $n=200$ cells for each individual. Statistical significance was calculated using a one-tailed t-test. ${ }^{*},{ }^{* *},{ }^{* * *}$, ${ }^{* * * *}$ correspond to $p$-values $<0.05,0.01,0.001$, and 0.0001 , respectively.

Fig 3. INTS13 associates with the INTS4/9/11 heterotrimer. a Yeast two hybrid screening for interactors of INTS13. Haploid strains of yeast expressing each individual 
939 Integrator subunit fused to the Gal4 activating domain (AD) were mated with strains 940 expressing INTS13 fused to the DNA binding domain (BD). The first and third panels 941 show mated yeast grown on selective media as a positive control (media lacking leucine, 942 tryptophan, and uracil). The remaining panels are of yeast plated on restrictive media 943 additionally lacking histidine to test for protein-protein interactions and with 3AT (3-amino944 1,2,4-triazole) to test the strength of the interaction. No interaction was observed between 945 INTS13 and any other individual subunit (second panel). When INTS9 and INTS11 were 946 expressed in trans with INTS13, there was a positive interaction with INTS4 (fourth panel).

947 b Point mutants of INTS4, INTS9, and INTS11 known to disrupt their interactions were 948 used in yeast two hybrid to demonstrate that the formation of the INTS4/9/11 heterotrimer 949 is necessary for INTS13 association. General locations of the mutations are indicated 950 with arrows on the schematic. INTS13 is fused to the activating domain in the rest of the yeast two hybrid assays shown in this study. c SDS PAGE gel of fractions from gel 952 filtration column of co-expressed full-length INTS4, INTS9, INTS11 and INTS13. The sample was first purified by nickel affinity chromatography. INTS4 carried an N-terminal His-tag, while the other three proteins were untagged. The positions of the fractions are indicated at the top of the gel. $\mathbf{d}$ Consecutive 200-amino acid truncations of INTS13 were tested for interaction with INTS4/9/11. In modified yeast two-hybrid assays, the only positive interactions seen are full length INTS13 and the last $\sim 200$ residues, 501-706. The patient mutations are marked on the schematic and occur in this region. Loss of interaction with INTS4/9/11 was observed using the mutant versions of INTS13.

Fig 4. OFD2 mutations lead to conserved disruption of interactions between the INTS10/13/14 module and the remainder of the Integrator complex. a Immunoprecipitation of FLAG-INTS13 from nuclear extract derived from HEK-293T cells stably expressing FLAG epitope tagged human INTS13-wild type (wt), Family 1 INTS13 mutant (F1), or Family 2 INTS13 mutant (F2). Nuclear extract derived from naïve HEK293T cells not expressing any FLAG epitope was used as a control. Left panel: IP-LC/MS was done for each sample, and quantitation is shown as a heat map. The color scale 
969

970

971

972

973

974

975

976

977

978

979

980

981

982

983

984

985

986

987

988

989

990

991

992

993

994

995

996

997

998

panel: Western blot analysis of either nuclear extract input or anti-FLAG immunoprecipitation using human Integrator antibodies. b Results shown are from parallel experiments conducted in a manner identical with panel (a) with the exception that nuclear extract was derived from Drosophila S2 cells expressing FLAG-dINTS13-wt or FLAG-tagged dINTS13 proteins where patient mutations were introduced into homologous regions of the Drosophila INTS13 cDNA. c Modified yeast two hybrid analysis where human INTS14 is expressed as a Gal4-DNA binding domain fusion, human INTS13 wt or mutant is expressed as a Gal4-Activation domain fusion, and human INTS10 is expressed in trans. Dilutions of yeast are plated on both permissive (left) and restrictive (right) media. Empty activation domain vector is used as a negative control. d Schematic of the Integrator $\mathrm{CM}$ associating with the remainder of Integrator subunits that is bridged to the INTS10/13/14 module through the C-terminus of INTS13, which is disrupted by OFD2 mutations.

Fig 5. INTS13 depletion leads to a loss of primary cilia and broad transcriptional perturbation of ciliary genes. a Western blot of whole cell lysates from RPE cells treated with non-targeting control siRNA (Con.) or either of two siRNA targeting INTS13 (INTS131, INTS13-2). Lysates were probed for either INTS13 or tubulin as a loading control. b Immunofluorescence imaging of RPE cells treated with siRNAs as in panel (a) to visualize primary cilia (PC). DAPI staining in blue indicates the nuclei, $y$-tubulin staining is shown in red to visualize the basal body, and ARL13B staining is shown in green to visualize the ciliary axoneme. Representative images show disrupted primary cilia that occurred when INTS13 was targeted by RNAi. c Images from panel (b) were quantified for each condition and graphed as a percentage of the number of control-treated cells with PC (data are mean $+/-$ SD, con. $n=3785,13-1 n=2969,13-2 n=2675$. Raw calculations for each condition were divided by the control mean to adjust control to 1). Statistical significance was calculated using a one-way ANOVA with multiple comparisons. d RNA sequencing was done on siRNA treated RPE cells in triplicate. Genes with significant changes in expression are shown by color indicating the log2 fold change. e Results from panel (d) were used for gene ontology analysis: the top five significant results are shown for 
upregulated and downregulated genes. All five GO terms for upregulated genes are related to cilia. f Quantitative RT-PCR validating three ciliary genes with expression

1001 changes identified using RNA-seq. Results are quantified from independent biological

1002 replicates ( $n=3$, data are mean $+/-S D$ ). Statistical significance was calculated using 1003 multiple Student t-tests with a Bonferroni correction. ${ }^{*},{ }^{* *},{ }^{* * *},{ }^{* * * *}$ correspond to $\mathrm{p}$-values 1004 $<0.05,0.01,0.001$, and 0.0001 , respectively.

Fig 6. INTS13 is required for embryonic development and ciliogenesis in Xenopus laevis. a In comparison to control embryos, morpholino (MO)-mediated knock down of INTS13 in Xenopus leads to severe developmental defects including a small head, big belly, short and down curved body axes, cardiac edema and massive developmental delay. $\mathbf{b}$ In situ hybridization using a specific probe for the multi-ciliated cell (MCC) marker ccdc 9 shows a reduction in ccdc 9 expression in MCC of morphants compared to control embryos. c Whole mount antibody staining for acetylated $\alpha$-tubulin shows a punctate pattern similar to $c c d c 9$. The puncta have a more condensed and stronger signal in control embryos. A higher magnification view of the MCC shows marked reduction in cilia compared to controls. d Western blot showing that the INTS13 MO was able to deplete INTS13 protein in Xenopus embryos. e Low magnification SEM imaging of Xenopus MCC at embryonic stage 28. MCCs of a control embryo possess multiple, long cilia, whereas the morphant MCCs display fewer cilia per cell which appear to be disorganized. f High magnification SEM imaging of Xenopus MCC at embryonic stage 28. Ciliary length seems unperturbed in morphant embryos, while the average number of cilia on individual MCCs is clearly reduced by INTS13 knockdown. The average MCC in morphants have less than half of the number of cilia per cell compared to control, as shown in the bar graph. $\mathbf{g}$ The ultrastructure of the cilia in morphants appears to show a normal $9+2$ structure as imaged by TEM. ${ }^{*},{ }^{* *},{ }^{* * *}$, correspond to p-values < 0.05, 0.01, 0.001, and 0.0001 , respectively. 
1029 phenotype and orofacial dysmorphisms at different ages including: a-c Bilateral cleft lip and palate (corrected after multiple surgical operations), hypertelorism, broad nasal bridge, flat philtrum, dental abnormalities, low set ear and rough and sparse hair. d 1032 Crowded optic disc. m Clinodactyly of toes. e-I Photographs of affected individual II.5 of 1033 Family 1. e-g Bilateral cleft lip and palate, hypertelorism, broad nasal bridge, flat philtrum, 1034 dental abnormalities. $\mathbf{h}$ Crowded optic disc. $\mathbf{i}$ Low set ear and rough and sparse hair. j, $\mathbf{h}$ 1035 Broad and mild brachydactyly and single palmar creases. I Clinodactyly of toes. $\mathbf{n}$ 1036 Pedigree of Family I. The genotypes for the corresponding mutation are indicated below each individual. The c.2004delA mutation segregated with the disease in this family. $(+/-$ ) denotes the heterozygous alleles, and (-/-) denotes the homozygous mutant alleles. 0v Photographs of affected individuals II.2 and II.4 of Family 2 at 27 and 20 years old, respectively. w Pedigree of Family 2. The genotypes for the corresponding mutation are shown below each individual which confirm the segregation of the c.1955C>T mutation in this family. $(+/+)$ denotes the homozygous wildtype alleles.

1043 Supplemental Fig 2. The C-terminus of INTS13 interacts with the Integrator 1044 Cleavage Module. a Expanded modified yeast two hybrid assay from Fig 2a. Included here are tests where INTS11 or INTS9 were expressed in trans along with INTS13binding domain and crossed with each Integrator subunit. No positive interactions were seen. b Left: Modified yeast two hybrid with INTS9 fused to the binding domain and INTS11 expressed in trans mated with yeast expressing each Integrator subunit fused to the activating domain to reconfirm that INTS4 interacts with the INTS9/11 heterodimer.

1050 Right: The same experiment with the addition of INTS4 expressed in trans to show that 1051 INTS13 interacts with the INTS4/9/11 heterotrimer. c Smaller portions (approximately 20 amino acids) of the INTS13 protein were removed from the necessary region for interaction shown in Fig 2d to determine that residues 577-706 were sufficient to interact with INTS4/9/11.

1055

Supplemental Fig 3. INTS13 interacts with INTS10/14. a Partial protein alignment of 1057 the C-terminus of human and Drosophila INTS13 to show the orthologous mutations made to recapitulate the patient mutations in Drosophila cells. The location and altered residues are marked in brown for Family 1 and in green for Family 2 . b Modified yeast 
1060 two hybrid testing the interaction between INTS10/13/14. Left: INTS14 fused to the 1061 binding domain was crossed with each Integrator subunit fused to the activating domain, 1062 and no positive interaction was seen on selective media. Right: INTS13 fused to the 1063 activating domain was crossed with yeast strains expressing INTS14-binding domain with 1064 each Integrator subunit in trans. A positive interaction was seen when INTS10 was 1065 expressed in trans.

1066

Supplemental Fig 4. Significant overlap in gene expression changes between two 1068 INTS13-targeting siRNAs. a Venn diagrams show the number of significantly 1069 upregulated or $\mathbf{b}$ downregulated genes for siRNA 13-1 and 13-2, and the genes in common between the two siRNAs as determined by RNA-seq.

1071

Supplemental Fig 5. Expression of ints13 during Xenopus development. a Detailed expression pattern of ints13 in Xenopus embryos analyzed by WISH and whole mount immunostaining. ints13 has both maternal and zygotic contribution. At 4 cell stage, ints13 transcripts are localized within the animal half. At stage 10, its expression covers the whole embryo except the blastopore. At stage 17, ints13 is observed in neural tube and neural folds. b Lateral view, anterior is right stage 22 ints13 starts to be enriched in brain, branchial arches (ba), pronephros (pn), eye vesicles (ev), ear (e), skin cells (sk) and somites (so). Lateral view, anterior is right of the embryo at stage 28, ints13 expression is also detected in ear (e), skin cells (sk) and pronephros (pn). Correlation of transcripts localization of ints 13 protein at stage 28 by wholemount embryo antibody staining using a custom polyclonal INTS13 (a-M) antibody, Lateral view, anterior is left.

Table 1. Clinical phenotypes of the affected individuals. (n.d) not determined, (+) affirmative, (-) negative, (M) male and (F) female. 


\section{References}

1094

1095

1. Lee JE, Gleeson JG. A systems-biology approach to understanding the ciliopathy disorders.

1096 Genome Med 3, 59 (2011).

1097

1098

1099

1100

1101

1102

1103

1104

1105

1106

1107

1108

1109

1110

1111

1112

1113

1114

1115

1116

1117

1118

1119

1120

1121

1122

1123

1124

1125

1126

2. Lee JE, Gleeson JG. Cilia in the nervous system: linking cilia function and neurodevelopmental disorders. Curr Opin Neurol 24, 98-105 (2011).

3. Raidt J, et al. Ciliary beat pattern and frequency in genetic variants of primary ciliary dyskinesia. Eur Respir J 44, 1579-1588 (2014).

4. Waters AM, Beales PL. Ciliopathies: an expanding disease spectrum. Pediatr Nephrol 26, 10391056 (2011).

5. Arts HH, Knoers NV. Current insights into renal ciliopathies: what can genetics teach us? Pediatr Nephrol 28, 863-874 (2013).

6. Estrada-Cuzcano A, Roepman R, Cremers FP, den Hollander Al, Mans DA. Non-syndromic retinal ciliopathies: translating gene discovery into therapy. Hum Mol Genet 21, R111-124 (2012).

7. Forsythe E, Beales PL. Bardet-Biedl syndrome. Eur J Hum Genet 21, 8-13 (2013).

8. Alexiev BA, Lin X, Sun CC, Brenner DS. Meckel-Gruber syndrome: pathologic manifestations, minimal diagnostic criteria, and differential diagnosis. Arch Pathol Lab Med 130, 1236-1238 (2006).

9. Badano JL, Mitsuma N, Beales PL, Katsanis N. The ciliopathies: an emerging class of human genetic disorders. Annu Rev Genomics Hum Genet 7, 125-148 (2006).

10. Baker K, Beales PL. Making sense of cilia in disease: the human ciliopathies. Am J Med Genet $C$ Semin Med Genet 151C, 281-295 (2009).

11. Ferkol TW, Leigh MW. Ciliopathies: the central role of cilia in a spectrum of pediatric disorders. $J$ Pediatr 160, 366-371 (2012). 
12. Davis EE, Katsanis N. The ciliopathies: a transitional model into systems biology of human genetic disease. Curr Opin Genet Dev 22, 290-303 (2012).

1130

1131

1132

1133

1134

1135

1136

1137

1138

1139

1140

1141

1142

1143

1144

1145

1146

1147

1148

1149

1150

1151

1152

1153

1154

1155

1156

1157

1158

1159

1160

1161

1162

1163

1164

1165

13. Leigh MW, et al. Clinical and genetic aspects of primary ciliary dyskinesia/Kartagener syndrome. Genet Med 11, 473-487 (2009).

14. Thauvin-Robinet $\mathrm{C}$, et al. The oral-facial-digital syndrome gene C2CD3 encodes a positive regulator of centriole elongation. Nat Genet 46, 905-911 (2014).

15. Gurrieri F, Franco B, Toriello H, Neri G. Oral-facial-digital syndromes: review and diagnostic guidelines. Am J Med Genet A 143A, 3314-3323 (2007).

16. Ferrante $\mathrm{Ml}$, et al. Convergent extension movements and ciliary function are mediated by ofd1, a zebrafish orthologue of the human oral-facial-digital type 1 syndrome gene. Hum Mol Genet 18, 289-303 (2009).

17. Thauvin-Robinet C, et al. OFD1 mutations in males: phenotypic spectrum and ciliary basal body docking impairment. Clin Genet 84, 86-90 (2013).

18. Thomas S, et al. TCTN3 mutations cause Mohr-Majewski syndrome. Am J Hum Genet 91, 372378 (2012).

19. Shamseldin HE, et al. Mutations in DDX59 implicate RNA helicase in the pathogenesis of orofaciodigital syndrome. Am J Hum Genet 93, 555-560 (2013).

20. Adly N, Alhashem A, Ammari A, Alkuraya FS. Ciliary genes TBC1D32/C6orf170 and SCLT1 are mutated in patients with OFD type IX. Hum Mutat 35, 36-40 (2014).

21. Franco B, Thauvin-Robinet C. Update on oral-facial-digital syndromes (OFDS). Cilia 5, 12 (2016).

22. Lopez E, et al. C5orf42 is the major gene responsible for OFD syndrome type VI. Hum Genet 133, 367-377 (2014).

23. Ferrante $\mathrm{MI}$, et al. Identification of the gene for oral-facial-digital type I syndrome. Am J Hum Genet 68, 569-576 (2001).

24. Roberson EC, et al. TMEM231, mutated in orofaciodigital and Meckel syndromes, organizes the ciliary transition zone. J Cell Biol 209, 129-142 (2015). 
25. Baillat D, Hakimi MA, Naar AM, Shilatifard A, Cooch N, Shiekhattar R. Integrator, a multiprotein mediator of small nuclear RNA processing, associates with the C-terminal repeat of RNA polymerase II. Cell 123, 265-276 (2005).

26. Baillat $D$, Wagner EJ. Integrator: surprisingly diverse functions in gene expression. Trends Biochem Sci 40, 257-264 (2015).

27. Gardini A, et al. Integrator Regulates Transcriptional Initiation and Pause Release following Activation. Mol Cell 56, 128-139 (2014).

1176

1177

1178

1179

1180

1181

1182

1183

1184

1185

1186

1187

1188

1189

1190

1191

1192

1193

1194

1195

1196

1197

1198

1199

1200

1201

1202

28. Skaar JR, et al. The Integrator complex controls the termination of transcription at diverse classes of gene targets. Cell Res 25, 288-305 (2015).

29. Stadelmayer B, et al. Integrator complex regulates NELF-mediated RNA polymerase II pause/release and processivity at coding genes. Nat Commun 5, 5531 (2014).

30. Albrecht TR, Wagner EJ. snRNA 3' end formation requires heterodimeric association of integrator subunits. Mol Cell Biol 32, 1112-1123 (2012).

31. Callebaut I, Moshous D, Mornon JP, de Villartay JP. Metallo-beta-lactamase fold within nucleic acids processing enzymes: the beta-CASP family. Nucleic Acids Res 30, 3592-3601 (2002).

32. Mandel CR, et al. Polyadenylation factor CPSF-73 is the pre-mRNA 3'-end-processing endonuclease. Nature 444, 953-956 (2006).

33. Albrecht TR, et al. Integrator subunit 4 is a 'Symplekin-like' scaffold that associates with INTS9/11 to form the Integrator cleavage module. Nucleic Acids Res 46, 4241-4255 (2018).

34. Ezzeddine $\mathrm{N}$, et al. A subset of Drosophila integrator proteins is essential for efficient U7 snRNA and spliceosomal snRNA 3'-end formation. Mol Cell Biol 31, 328-341 (2011).

35. Lai F, Gardini A, Zhang A, Shiekhattar R. Integrator mediates the biogenesis of enhancer RNAs. Nature 525, 399-403 (2015).

36. Rubtsova MP, et al. Integrator is a key component of human telomerase RNA biogenesis. Sci Rep 1203 
1204 37. Elrod ND, et al. The Integrator Complex Attenuates Promoter-Proximal Transcription at ProteinCoding Genes. Mol Cell 76, 738-752 e737 (2019).

1206

1207

1208

1209

1210

1211

1212

1213

1214

1215

1216

1217

1218

1219

1220

1221

1222

1223

1224

1225

1226

1227

1228

1229

1230

1231

1232

1233

1234

1235

1236

1237

1238

1239

1240

1241

38. Tatomer DC, et al. The Integrator complex cleaves nascent mRNAs to attenuate transcription. Genes Dev 33, 1525-1538 (2019).

39. Jodoin JN, et al. Nuclear-localized Asunder regulates cytoplasmic dynein localization via its role in the integrator complex. Mol Biol Cell 24, 2954-2965 (2013).

40. Sorokin S. Centrioles and the formation of rudimentary cilia by fibroblasts and smooth muscle cells. J Cell Biol 15, 363-377 (1962).

41. Jodoin JN, et al. The snRNA-processing complex, Integrator, is required for ciliogenesis and dynein recruitment to the nuclear envelope via distinct mechanisms. Biol Open 2, 1390-1396 (2013).

42. Breslow DK, et al. A CRISPR-based screen for Hedgehog signaling provides insights into ciliary function and ciliopathies. Nat Genet 50, 460-471 (2018).

43. Sakai N, Nakakita N, Yamazaki Y, Ui K, Uchinuma E. Oral-facial-digital syndrome type II (Mohr syndrome): clinical and genetic manifestations. J Craniofac Surg 13, 321-326 (2002).

44. Bonnard C, et al. Mutations in IRX5 impair craniofacial development and germ cell migration via SDF1. Nat Genet 44, 709-713 (2012).

45. Duquette A, Brais B, Bouchard JP, Mathieu J. Clinical presentation and early evolution of spastic ataxia of Charlevoix-Saguenay. Mov Disord 28, 2011-2014 (2013).

46. Synofzik $M$, et al. Autosomal recessive spastic ataxia of Charlevoix Saguenay (ARSACS): expanding the genetic, clinical and imaging spectrum. Orphanet J Rare Dis 8, 41 (2013).

47. Lee LA, et al. Drosophila genome-scale screen for PAN GU kinase substrates identifies Mat89Bb as a cell cycle regulator. Dev Cell 8, 435-442 (2005).

48. Stebbings L, Grimes BR, Bownes M. A testis-specifically expressed gene is embedded within a cluster of maternally expressed genes at 89B in Drosophila melanogaster. Dev Genes Evol 208, 523-530 (1998). 
49. Anderson MA, Jodoin JN, Lee E, Hales KG, Hays TS, Lee LA. Asunder is a critical regulator of dynein-dynactin localization during Drosophila spermatogenesis. Mol Biol Cell 20, 2709-2721 (2009).

50. Chen J, et al. An RNAi screen identifies additional members of the Drosophila Integrator complex and a requirement for cyclin C/Cdk8 in snRNA 3'-end formation. RNA 18, 2148-2156 (2012).

51. Boon $\mathrm{M}$, et al. Primary ciliary dyskinesia: critical evaluation of clinical symptoms and diagnosis in patients with normal and abnormal ultrastructure. Orphanet J Rare Dis 9, 11 (2014).

52. Erle DJ, Sheppard D. The cell biology of asthma. J Cell Biol 205, 621-631 (2014).

53. Livraghi A, Randell SH. Cystic fibrosis and other respiratory diseases of impaired mucus clearance. Toxicol Pathol 35, 116-129 (2007).

54. Turner J, Jones CE. Regulation of mucin expression in respiratory diseases. Biochem Soc Trans 37, 877-881 (2009).

55. Wallmeier J, et al. Mutations in CCNO result in congenital mucociliary clearance disorder with reduced generation of multiple motile cilia. Nat Genet 46, 646-651 (2014).

56. Sabath K, Staubli ML, Marti S, Leitner A, Moes M, Jonas S. INTS10-INTS13-INTS14 form a functional module of Integrator that binds nucleic acids and the cleavage module. Nat Commun 11, 3422 (2020).

57. Wu Y, Albrecht TR, Baillat D, Wagner EJ, Tong L. Molecular basis for the interaction between Integrator subunits IntS9 and IntS11 and its functional importance. Proc Natl Acad Sci U S A 114, 4394-4399 (2017).

58. Chen J, Waltenspiel B, Warren WD, Wagner EJ. Functional analysis of the integrator subunit 12 identifies a microdomain that mediates activation of the Drosophila integrator complex. $J$ Biol Chem 288, 4867-4877 (2013).

59. Blacque OE, et al. Functional genomics of the cilium, a sensory organelle. Curr Biol 15, 935-941 (2005).

60. Brooks ER, Wallingford JB. Multiciliated cells. Curr Biol 24, R973-982 (2014). 
61. Wallingford JB. Planar cell polarity signaling, cilia and polarized ciliary beating. Curr Opin Cell Biol 22, 597-604 (2010).

62. Hayes JM, et al. Identification of novel ciliogenesis factors using a new in vivo model for mucociliary epithelial development. Dev Biol 312, 115-130 (2007).

63. Barbieri E, et al. Targeted Enhancer Activation by a Subunit of the Integrator Complex. Mol Cell 71, 103-116 e107 (2018).

64. Walker JR, Corpina RA, Goldberg J. Structure of the Ku heterodimer bound to DNA and its implications for double-strand break repair. Nature 412, 607-614 (2001).

65. Oegema R, et al. Human mutations in integrator complex subunits link transcriptome integrity to brain development. PLoS Genet 13, e1006809 (2017).

66. Del Viso F, et al. Congenital Heart Disease Genetics Uncovers Context-Dependent Organization and Function of Nucleoporins at Cilia. Dev Cell 38, 478-492 (2016).

67. Fakhro KA, et al. Rare copy number variations in congenital heart disease patients identify unique genes in left-right patterning. Proc Natl Acad Sci U S A 108, 2915-2920 (2011).

68. Schmidt KN, Kuhns S, Neuner A, Hub B, Zentgraf H, Pereira G. Cep164 mediates vesicular docking to the mother centriole during early steps of ciliogenesis. J Cell Biol 199, 1083-1101 (2012).

69. Galati DF, Sullivan KD, Pham AT, Espinosa JM, Pearson CG. Trisomy 21 Represses Cilia Formation and Function. Dev Cell 46, 641-650 e646 (2018).

70. Homer N, Merriman B, Nelson SF. BFAST: an alignment tool for large scale genome resequencing. PLoS One 4, e7767 (2009).

71. Fitzgerald DJ, Berger P, Schaffitzel C, Yamada K, Richmond TJ, Berger I. Protein complex expression by using multigene baculoviral vectors. Nat Methods 3, 1021-1032 (2006).

72. Anderson AP, Luo X, Russell W, Yin YW. Oxidative damage diminishes mitochondrial DNA polymerase replication fidelity. Nucleic Acids Res 48, 817-829 (2020).

73. Jaworski E, Routh A. ClickSeq: Replacing Fragmentation and Enzymatic Ligation with ClickChemistry to Prevent Sequence Chimeras. Methods Mol Biol 1712, 71-85 (2018). 
bioRxiv preprint doi: https://doi.org/10.1101/2020.07.20.209130; this version posted July 24, 2020. The copyright holder for this preprint (which was not certified by peer review) is the author/funder. All rights reserved. No reuse allowed without permission. 


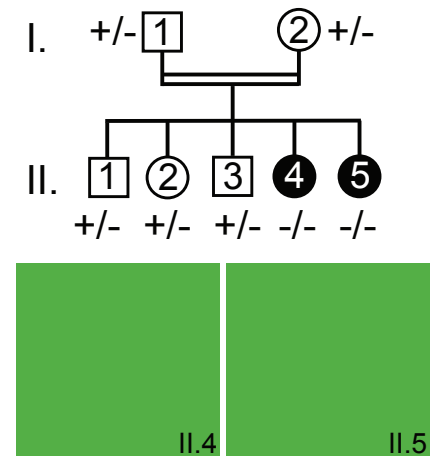

Family 2

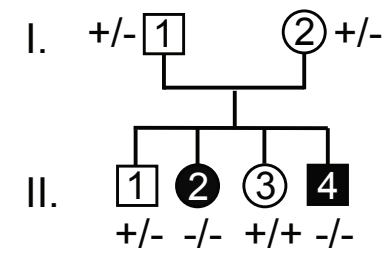

b

Homozygosity Mapping

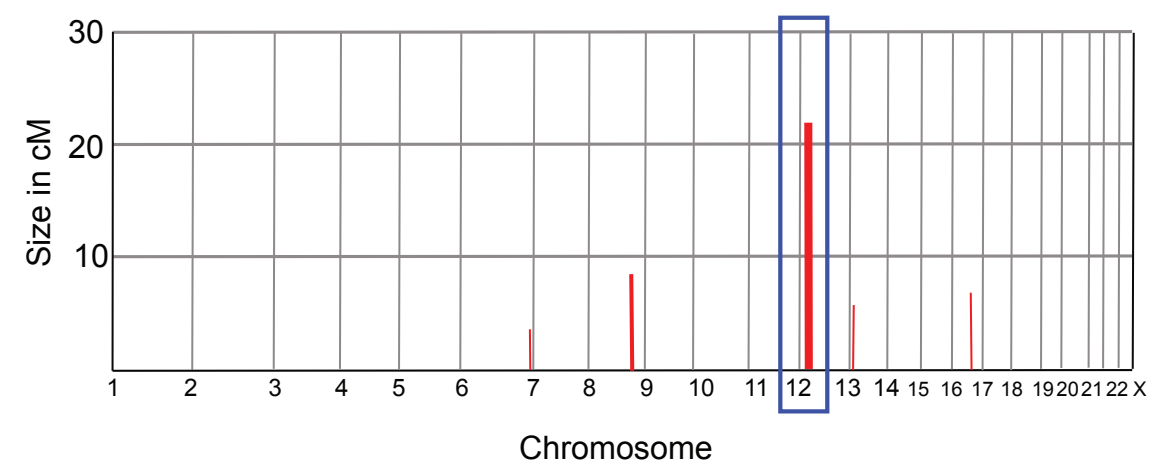

C
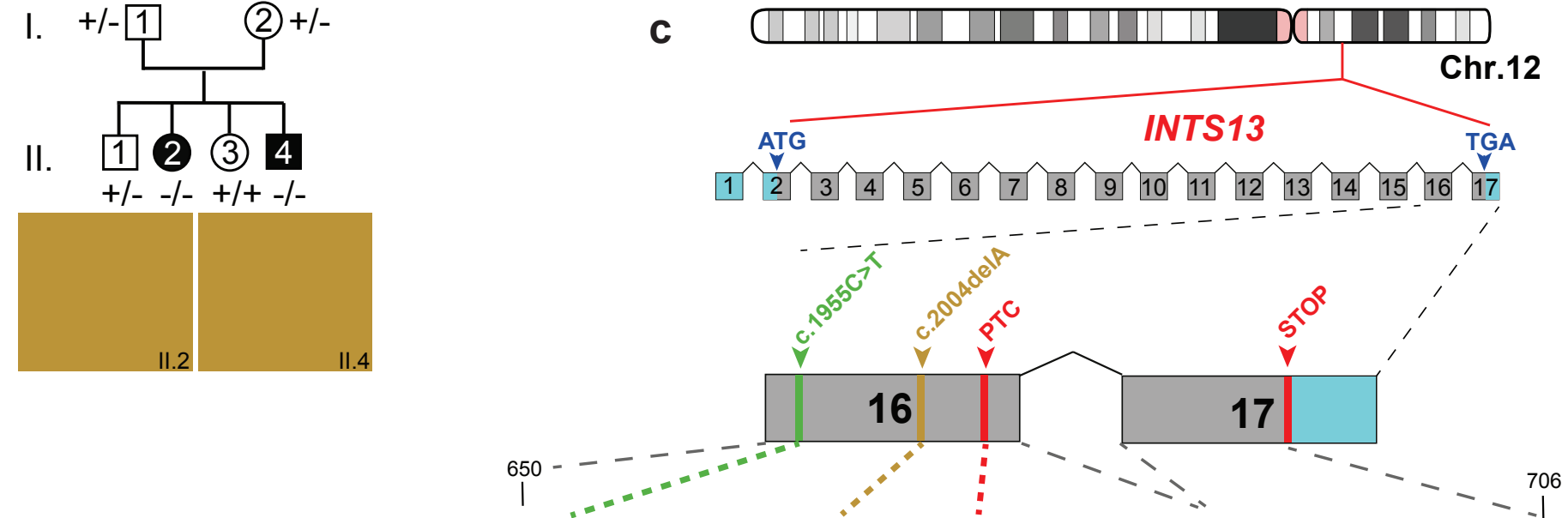

INTS13-WT .. GPVŚLLSLWSNRINTANSRKHQEFAGRLLNSVNNRAELYQHLKEENGMETTENGKASRQ

Family 1 .. GPVSLLSLWSNRINTANSR NIRNLLDV

Family 2 .. GPVLLLSLWSNRINTANSRKHQEFAGRLNSVNNRAELYQHLKEENGMETTENGKASRQ

\section{d}

S652L $650 \downarrow$

Human

$\mathrm{K} 668 \mathrm{Nfs} * 9$

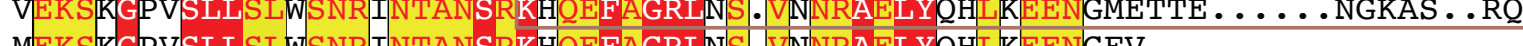

Chicken VEKAKGPMSLLSLWSNR INTANSRKHOEF I GRLNS. VNNKAEL YOHL KEENGMETTE . . . . . . NGKTG . RO RO

Frog AEKSKGPMSLLSLWSSRINTANSRKHOEFVGRLNS. VNNKAELYOHL KEENGGEGVE $\ldots \ldots \ldots$ NGKAS . RQ RQ

Fish PEKSKGPVSLLALWTNRITQANSRKHQEFAGRLSS. VNNKAELYQHLKEENGMDVHE . . . . . . NGKAT . . R.

Fly RMSSCGQRSLLDIISSA. ERSOSNKRLDFSGRLCTPLGQVAKLYPDEGTKDKDTVTTGASITPNVKEESVRS

Fig 1. Identification of two families segregating recessive mutations in INTS13. a Pedigree of Family 1 (top): affected individuals (II.4 and II.5) were born to first-cousin parents (I.1 and I.2) with three unaffected children (II.1-II.3). Double lines indicate a consanguineous marriage. Pedigree of Family 2 (bottom): affected individuals (II.2 and II.4) were born to parents with two unaffected children (II.1 and II.3). b Homozygosity mapping of Family 1 delineated five candidate loci totaling $46 \mathrm{cM}$ on chromosomes $6,8,12,13$, and 16. c Schematic representation of human INTS13 located on chromosome 12 (ch12p13.2-p11.22) consisting of 17 exons. Locus capture followed by massive parallel sequencing identified a homozygous frameshift mutation as a disease-causing mutation in Family 1 . The c.2004deIA (p.K668Nfs ${ }^{*}$ ) mutation is caused by a single base pair deletion in exon 16 (marked in brown), resulting in a frame shift and premature termination codon (PTC) which alters nine amino acids (marked in red) and deletes the last 31. In Family 2, the c.1955C >T (S652L) mutation is caused by a single base pair substitution in exon 16 (marked in green). d Amino acid alignment of the C-terminus of the INTS13 protein shows that both mutations occur at highly conserved residues. Red and yellow shading indicates regions of conservation. 

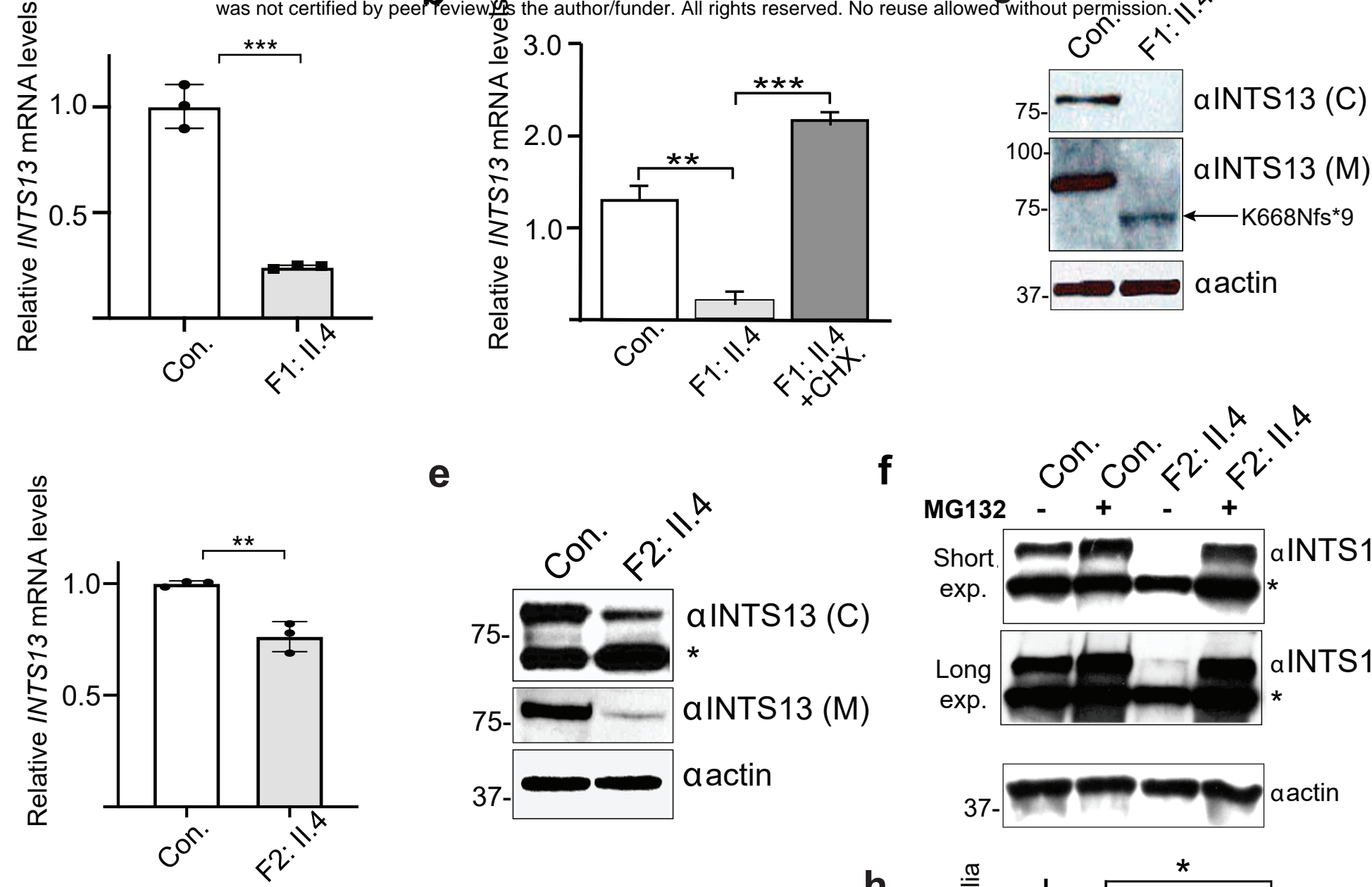

e

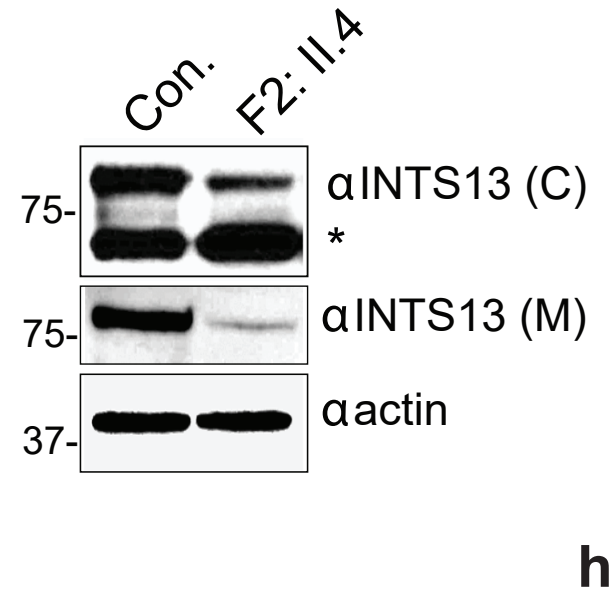

g

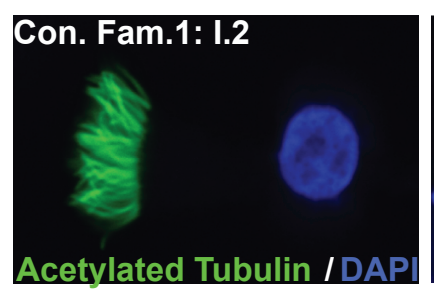

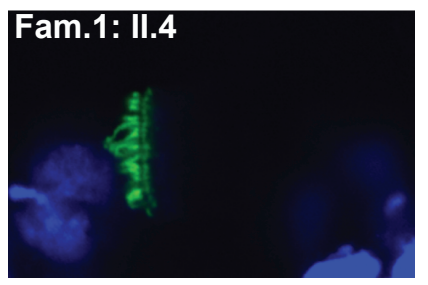

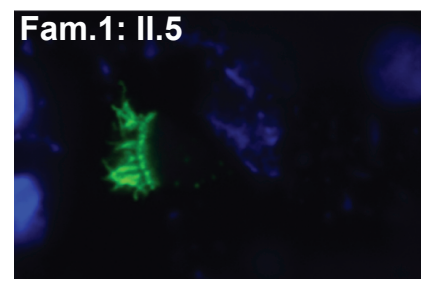

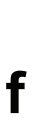

\section{MG132}

h
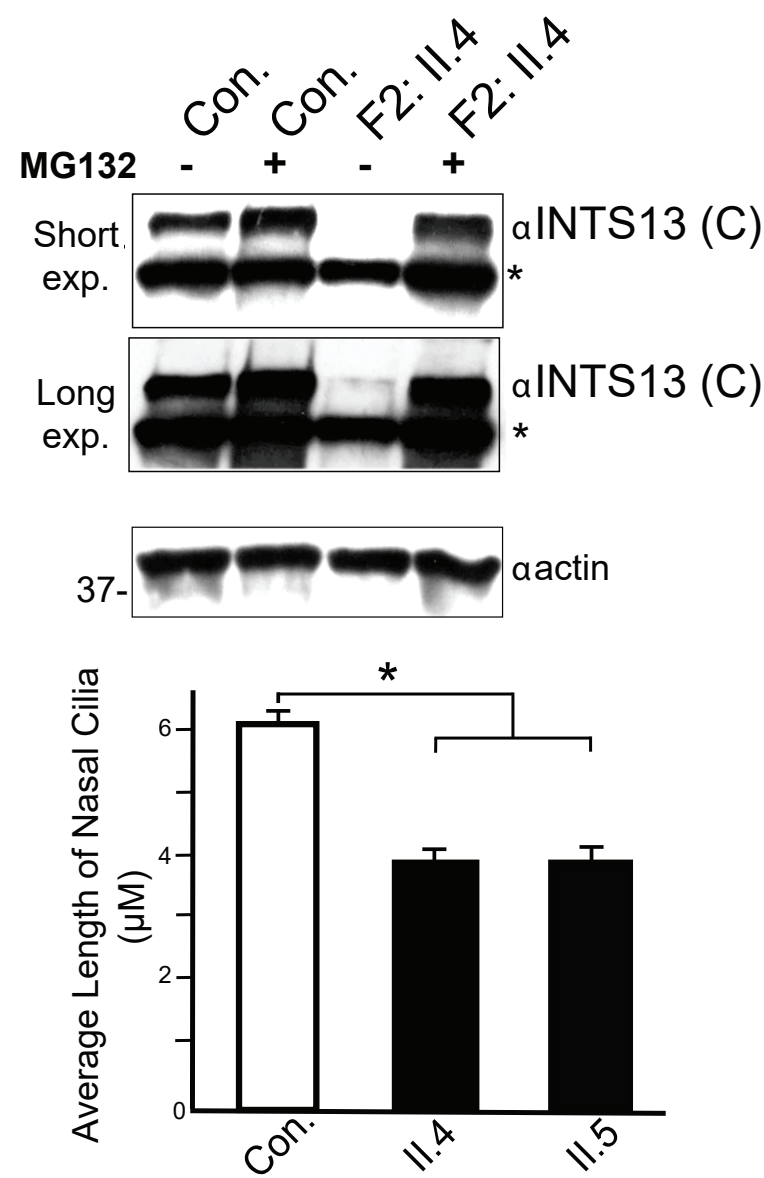

Fig 2. Characterization of Loss-of-function INTS13 mutations in patient cells. a RT-qPCR shows that endogenous INTS13 transcript levels were significantly reduced relative to control cells in primary dermal fibroblasts of affected individual II. 4 from Family 1 . Data are mean $+/-S D, n=3, p<0.001$, two-tailed unpaired t-test. b Primary dermal fibroblasts from individual II.4 from Family 1 were treated with $100 \mathrm{~g} / \mathrm{ml}$ cycloheximide (CHX). Following treatment, RT-qPCR was done which shows that the INTS13 mRNA level was increased in treated cells. c Western blot analysis of primary dermal fibroblasts of affected individual II.4 from Family 1 using two polyclonal antibodies designed against distinct regions of INTS13. No signal was detected in patient cells with the antibody directed against the C-terminus of INTS13 (C). A truncated protein was detected in patient cells by the antibody directed against the center of the protein (M). Actin was used as a loading control. $\mathbf{d}$ RT-qPCR shows that INTS13 transcript levels were significantly reduced in primary dermal fibroblast cells of affected individual II.4 from Family 2 relative to control cells. Data are mean $+/-S D, n=3, p<0.01$, two-tailed unpaired t-test. e Western blot analysis of primary dermal fibroblasts of affected individual II.4 from Family 2 shows reduced levels of INTS13 protein compared to control cells. $\mathbf{f}$ Western blot analysis of primary dermal fibroblasts from individual II.4 from Family 2 treated with MG132. Treated cells show a level of INTS13 protein comparable to control cells. * indicates a non-specific signal. g Multiciliated airway cells were obtained from nasal biopsies of three members of Family 1: the unaffected carrier mother (Con.) and the two affected children (II.4 and II.5). Probing for acetylated a-tubulin shows significantly shorter, less dense, and disorganized cilia in the affected individuals compared to the mother's cells. $\mathbf{h}$ Bar graph representing the average length of nasal cilia. Data are mean \pm s.e.m., $n \geq 31$ cells for each individual. ${ }^{*}, * * * * *, * * * *$ correspond to $p$-values $<0.05,0.01,0.001$, and 0.0001 , respectively. 


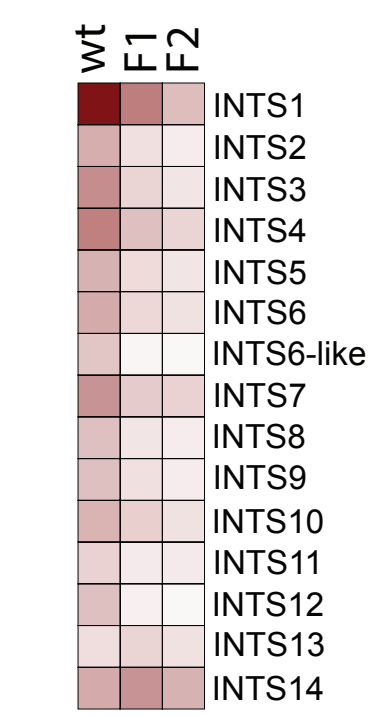

0.00 102.00 Normalized spectral counts

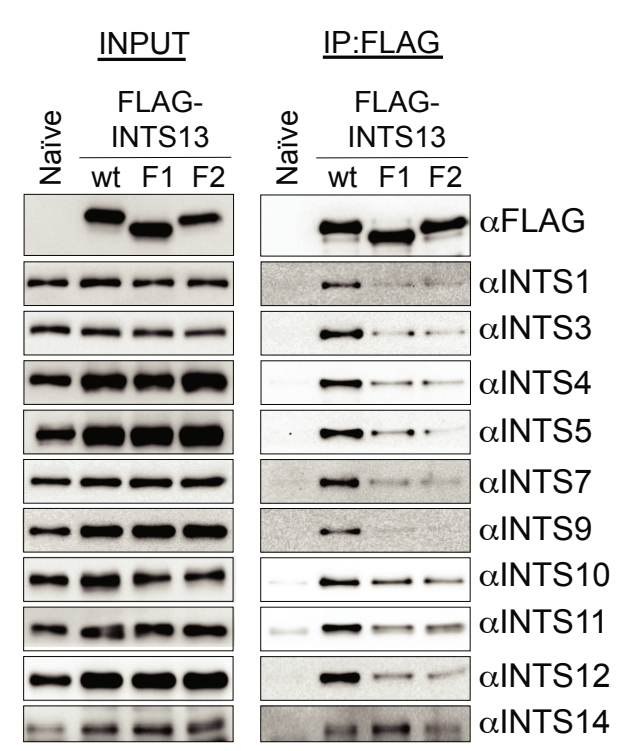

10

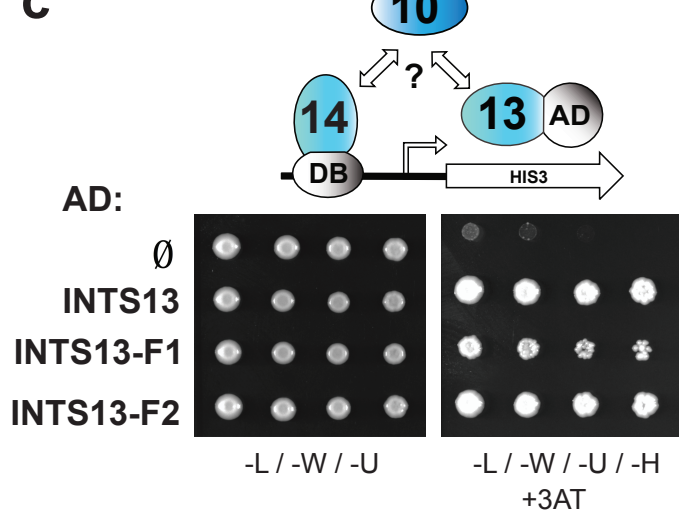

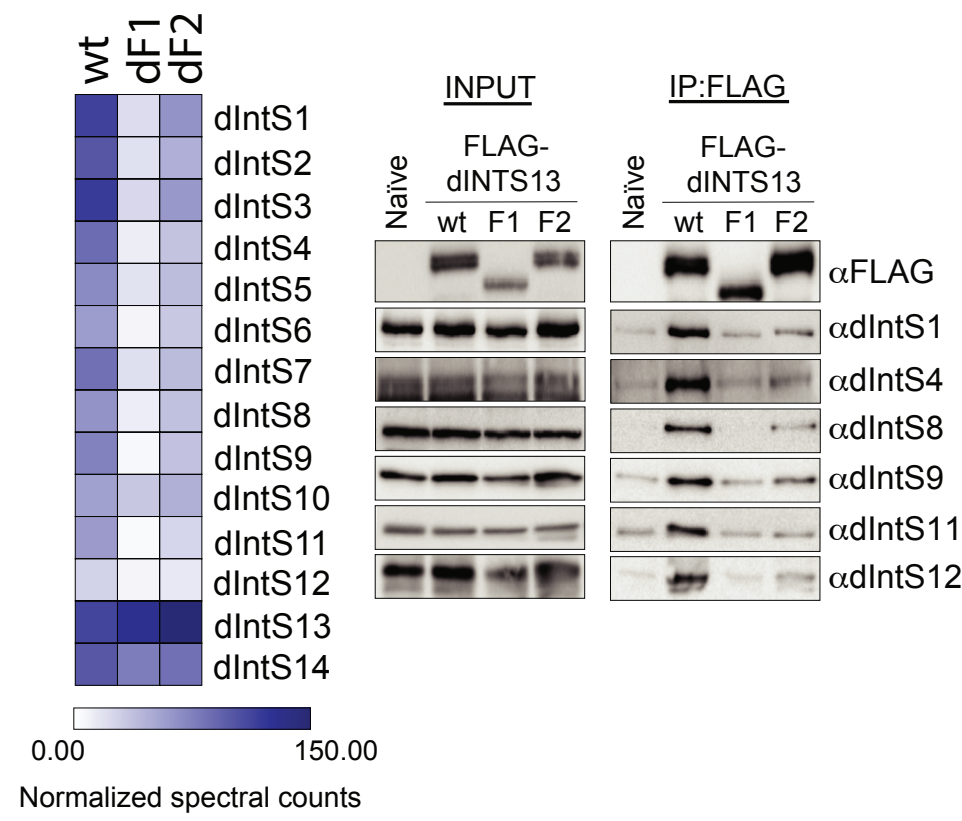

d

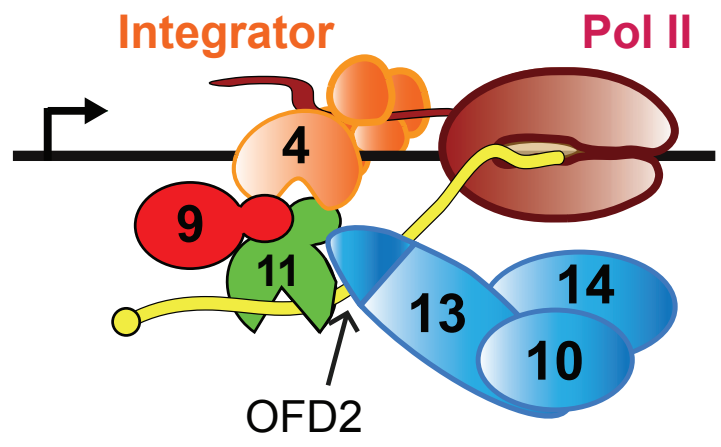

mutations

Fig 4. OFD2 mutations lead to conserved disruption of interactions between the INTS10/13/14 module and the remainder of the Integrator complex. a Immunoprecipitation of FLAG-INTS13 from nuclear extract derived from HEK-293T cells stably expressing FLAG epitope tagged human INTS13-wild type (wt), Family 1 INTS13 mutant (F1), or Family 2 INTS13 mutant (F2). Nuclear extract derived from naïve HEK-293T cells not expressing any FLAG epitope was used as a control. Left panel: IP-LC/MS was done for each sample, and quantitation is shown as a heat map. The color scale shown denotes the normalized spectral counts for each protein (see methods). Right panel: Western blot analysis of either nuclear extract input or anti-FLAG immunoprecipitation using human Integrator antibodies. b Results shown are from parallel experiments conducted in a manner identical with panel (a) with the exception that nuclear extract was derived from Drosophila S2 cells expressing FLAG-dINTS13-wt or FLAG-tagged dINTS13 proteins where patient mutations were introduced into homologous regions of the Drosophila INTS13 cDNA. c Modified yeast two hybrid analysis where human INTS14 is expressed as a Gal4-DNA binding domain fusion, human INTS13 wt or mutant is expressed as a Gal4-Activation domain fusion, and human INTS10 is expressed in trans. Dilutions of yeast are plated on both permissive (left) and restrictive (right) media. Empty activation domain vector is used as a negative control. d Schematic of the Integrator CM associating with the remainder of Integrator subunits that is bridged to the INTS10/13/14 module through the C-terminus of INTS13, which is disrupted by OFD2 mutations. 


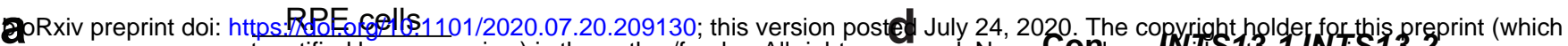

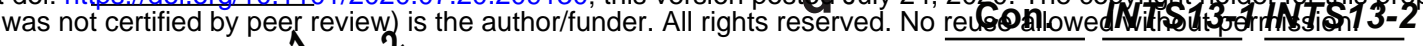
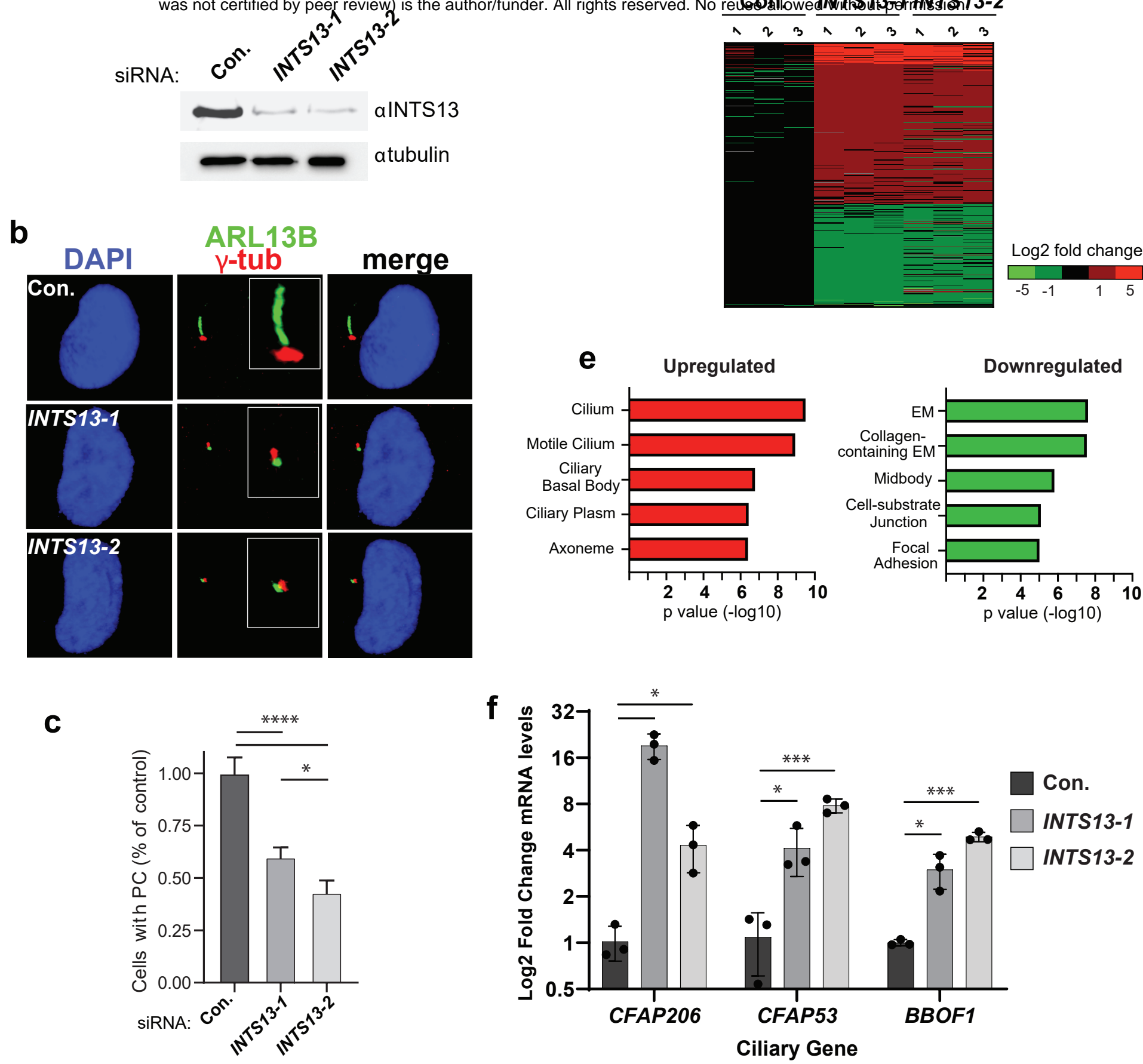

Fig 5. INTS13 depletion leads to a loss of primary cilia and broad transcriptional perturbation of ciliary genes. a Western blot of whole cell lysates from RPE cells treated with non-targeting control siRNA (Con.) or either of two siRNA targeting INTS13 (INTS13-1, INTS13-2). Lysates were probed for either INTS13 or tubulin as a loading control. b Immunofluorescence imaging of RPE cells treated with siRNAs as in panel (a) to visualize primary cilia (PC). DAPI staining in blue indicates the nuclei, $y$-tubulin staining is shown in red to visualize the basal body, and ARL13B staining is shown in green to visualize the ciliary axoneme. Representative images show disrupted primary cilia that occurred when INTS13 was targeted by RNAi. c Images from panel (b) were quantified for each condition and graphed as a percentage of the number of control-treated cells with PC (data are mean $+/$ - SD, con. $n=3785,13-1 n=2969,13-2 n=2675$. Raw calculations for each condition were divided by the control mean to adjust control to 1). Statistical significance was calculated using a one-way ANOVA with multiple comparisons with $p<0.05\left({ }^{*}\right)$ and $p<0.0001\left(^{* *}\right)$. d RNA sequencing was done on siRNA treated RPE cells in triplicate. Genes with significant changes in expression are shown by color indicating the log2 fold change. e Results from panel (d) were used for gene ontology analysis: the top five significant results are shown for upregulated and downregulated genes. All five GO terms for upregulated genes are related to cilia. $f$ Quantitative RT-PCR validating three ciliary genes with expression changes identified using RNA-seq. Results are quantified from independent biological replicates $(n=3$, data are mean $+/$ SD). Statistical significance was calculated using multiple Student T-tests with a Bonferroni correction, with $p<0.05\left({ }^{*}\right)$ and $p<0.001\left(^{* *}\right) .{ }^{*},{ }^{* *},{ }^{* * *},{ }^{* * *}$ correspond to $p$-values $<0.05,0.01,0.001$, and 0.0001 , respectively. 


\begin{tabular}{|c|c|c|c|c|}
\hline \multirow{2}{*}{$\begin{array}{l}\text { Clinical synopsis } \\
\text { Origin }\end{array}$} & \multicolumn{3}{|c|}{ Observations in probands } & \multirow[b]{2}{*}{ France } \\
\hline & Jordan & Jordan & France & \\
\hline Consanguinity & + & + & + & + \\
\hline Patient number (refer to pedigree) & II.4 & 11.5 & 11.2 & $\pi .4$ \\
\hline Year of birth & 2002 & 2005 & 1987 & 1994 \\
\hline Gender & $\mathrm{F}$ & $\mathrm{F}$ & $\mathrm{F}$ & $\mathrm{M}$ \\
\hline \multicolumn{5}{|l|}{ Oral } \\
\hline Cleft / high arched palate & + & + & - & - \\
\hline Tongue anomalies/hamartoma/lipoma & - & - & - & - \\
\hline Teeth abnormalities & + & + & - & - \\
\hline Micrognathia & + & + & - & - \\
\hline \multicolumn{5}{|l|}{ Craniofacial } \\
\hline Microcephaly & + & + & - & - \\
\hline Ocular hyperletorism & + & + & - & - \\
\hline Median cleft upper lip & Bilateral & Bilateral & - & - \\
\hline Broad nasal bridge & + & + & - & - \\
\hline Nasal tip anomalies & + & + & + & + \\
\hline Low set ears & + & + & - & - \\
\hline \multicolumn{5}{|l|}{ Hands } \\
\hline Syndactyly & - & - & - & - \\
\hline Clinodactyly & + & - & - & - \\
\hline Pre-Polydactyly & + & - & - & - \\
\hline Bifid/duplicated thumb & - & - & - & - \\
\hline \multicolumn{5}{|l|}{ Feet } \\
\hline Syndactyly & - & - & - & - \\
\hline Clinodactyly & + & - & - & - \\
\hline Polydactyly (pre-, post-axial) & - & - & - & - \\
\hline Bilateral hallux duplication & - & - & - & - \\
\hline \multicolumn{5}{|l|}{ Variable Findings } \\
\hline Lower stature/short humerus & - & - & + & + \\
\hline Pectus escavatum & - & - & - & - \\
\hline Absence of central incisors & - & - & - & - \\
\hline Supernumerary teeth & + & + & - & - \\
\hline Oral frenulae & - & - & - & - \\
\hline Long philtrum & + & + & - & - \\
\hline Thick septum & + & + & - & - \\
\hline Epiglottis anomaly & + & + & - & - \\
\hline Central Y-shaped metacarpa & - & - & - & - \\
\hline CNS abnormalities & - & - & + & + \\
\hline Speech abnormality & + & + & + & + \\
\hline Congenital heart defects & + & - & - & - \\
\hline Conductive hearing loss & + & + & - & - \\
\hline Epicanthal fold & + & + & - & - \\
\hline Respiratory tract infections and cough & + & + & - & - \\
\hline \multicolumn{5}{|l|}{ Unique features seen in present propositae } \\
\hline Alveolar pulmonary stenosis & - & + & - & - \\
\hline Omega shaped epiglottis & + & + & - & - \\
\hline Underdeveloped aryepiglottic folds & + & + & - & - \\
\hline Hypertrophied false vocal cords & + & + & nd & nd \\
\hline Presence of single central upper incisors & + & + & - & - \\
\hline Racemose angioma & + & + & - & - \\
\hline Crowded optic disc & + & + & nd & nd \\
\hline Myringosclerosis & + & + & nd & nd \\
\hline Adenoid hypertrophy & n.d. & + & nd & nd \\
\hline Opacification of mastoid and ethmoid air cells & + & n.d. & nd & nd \\
\hline Rough and sparse hair & + & + & + & + \\
\hline Bilateral simian creases & + & + & - & - \\
\hline Short \& broad fingers & + & + & + & + \\
\hline
\end{tabular}

